NBER WORKING PAPER SERIES

\title{
OWNING THE AGENT: \\ HOSPITAL INFLUENCE ON PHYSICIAN BEHAVIORS
}

\author{
Haizhen Lin \\ Ian M. McCarthy \\ Michael R. Richards \\ Christopher Whaley \\ Working Paper 28859 \\ http://www.nber.org/papers/w28859 \\ NATIONAL BUREAU OF ECONOMIC RESEARCH \\ 1050 Massachusetts Avenue \\ Cambridge, MA 02138 \\ May 2021, Revised September 2022
}

We thank participants and discussants at the American Economics Association Annual Meeting, the American Society of Health Economists Conference, the Southeastern Health Economics Study Group, and seminar participants at Johns Hopkins University, George Washington University, Indiana University, West Virginia University, and the University of Nevada, Las Vegas. This work began while Dr. Richards was a member of Vanderbilt University's Department of Health Policy. He is grateful to the department for its generous financial support and access to excellent data resources. This project was also supported in-part by grant numbers R00HS022431 from the Agency for Healthcare Research and Quality and 1K01AG061274 from the National Institute of Aging. The content is solely the responsibility of the authors and does not necessarily represent the official views of the Agency for Healthcare Research and Quality, the National Institute of Aging, or the National Bureau of Economic Research.

NBER working papers are circulated for discussion and comment purposes. They have not been peer-reviewed or been subject to the review by the NBER Board of Directors that accompanies official NBER publications.

(C) 2021 by Haizhen Lin, Ian M. McCarthy, Michael R. Richards, and Christopher Whaley. All rights reserved. Short sections of text, not to exceed two paragraphs, may be quoted without explicit permission provided that full credit, including $\odot$ notice, is given to the source. 
Owning the Agent: Hospital Influence on Physician Behaviors

Haizhen Lin, Ian M. McCarthy, Michael R. Richards, and Christopher Whaley

NBER Working Paper No. 28859

May 2021, Revised September 2022

JEL No. H51,I11,I18

\begin{abstract}
The organizational structure of U.S. health care has changed dramatically in recent years, with nearly half of physicians now employed by hospitals. This trend toward increasing vertical alignment between physicians and hospitals may alter physician behavior relative to physicians remaining in independent or group practices. We examine the effects of such vertical alignment using an instrumental variable strategy and a clinical context facilitating well-defined episodes of care. We find no significant or economically meaningful effect on total Medicare payments per episode, characterized by a large and statistically significant reduction in professional services claims, particularly among referrals to other non-integrated physicians. Acquiring hospitals ultimately capture more revenue following a physician practice acquisition; yet, the smaller overall bundle of care generates no net savings to Medicare due to location-based payment rules favorable to hospitals.

Haizhen Lin

Department of Business Economics

and Public Policy

Kelley School of Business

Indiana University

1309 East Tenth Street

Bloomington, IN 47405

and NBER

hzlin@indiana.edu

Ian M. McCarthy

Department of Economics

Emory University

Rich Memorial Building, Room 319

Atlanta, GA 30322

and NBER

immccar@emory.edu

Michael R. Richards

Baylor University

Department of Economics

One Bear Place \#98003

Waco, TX 76798

michael_richards@baylor.edu

Christopher Whaley

RAND Corporation

1776 Main Street

Santa Monica, CA 90401

cwhaley@rand.org
\end{abstract}

A data appendix is available at http://www.nber.org/data-appendix/w28859

GitHub Replication Files is available at https://github.com/imccart/physician-hospital-VI 


\section{Introduction}

Hospital and physician services constitute the two largest components of U.S. health expenditures and jointly accounted for nearly \$2.1 trillion in U.S. health spending in 2019 (55\% of total health expenditures). Physicians also serve as gatekeepers for hospital-based services by referring patients to specific providers. Hospitals as downstream firms therefore have clear incentives to influence physician behavior where possible to improve profitability (Gaynor \& Town, 2012; Gaynor et al., 2015; Post et al., 2018). Moreover, the opportunity to influence treatment patterns for financial gain exists so long as patients remain at an informational disadvantage and insurer monitoring of physician decisions is incomplete. ${ }^{1}$ Such strategic behaviors can ultimately impact prevailing competition within a given market and related outcomes, such as service prices, that are of consumer and regulatory interest. For the public insurer, Medicare, altered physician behavior can translate to higher spending for the same general bundle of care due to its standing policies regarding site of care payment differentials_-policies that are also a source of ongoing controversy and debate.

Traditional physician-hospital relationships rely heavily on informal arrangements and often incomplete incentive alignment, thereby limiting a hospital's direct control over its revenue streams. Hospital outright acquisitions of physician practices, however, have the potential to influence physician behaviors in at least two general ways. First, following integration, hospitals become the residual claimant for malleable income streams that are strongly shaped by physician decision-making, including physicians' directly provided services, complementary services (e.g., diagnostic tests), and referrals to other providers owned by the hospital (Kocher \& Sahni, 2011). Indirectly, hospitals may also influence physician referrals to other non-integrated providers. The ability of the hospital to profitably influence these separate but interdependent sources of revenue will depend on the amount of control that can be exercised over the newly acquired practice. Second, organizational structure is known to influence physician effort and revenue generation. Economists have long recognized the inherent trade-off between risk-spreading and incentivizing individual physician output when forming groups (Gaynor \& Gertler, 1995). In the context of practice acquisitions, vertical integration could disincentivize physician effort (i.e., billable activity) by joining the affected physician with a larger group of other hospital-employed physicians and diminishing the financial incentives tied to ownership of a practice. ${ }^{2}$ It is therefore an open empirical question as to how these different and potentially

\footnotetext{
${ }^{1}$ There is now a substantial literature showing that a large share of spending is not driven by patient preferences. See, for example, Wennberg \& Gittelsohn (1973), Skinner \& Fisher (1997), Wennberg et al. (2003), Baicker \& Chandra (2004), and Gottlieb et al. (2010), among others. More recently, Finkelstein et al. (2016) exploits patient migration to estimate that $50-60 \%$ of geographic variation in spending is due to supply-side factors. Molitor (2018) likewise uses cardiologists' migration patterns to show that $60-80 \%$ of the variation in treatment styles can be explained by characteristics of the local physician practice.

${ }^{2}$ In our context, practice ownership prior to being vertically integrated could be a sole proprietorship or a partnership
} 
countervailing forces within vertical structures ultimately shape the overall treatment intensity and spending belonging to a given episode of care and to total billable activity among acquired physicians. This paper aims to quantify hospitals' influence on physician behaviors. We consider hospital acquisitions of physician practices as movement toward greater monitoring and control by hospitals and possibly improved incentive alignment between hospitals and physicians.

Our analysis allows us to decompose the effects of vertical integration on physician behaviors into multiple dimensions. We accomplish this in-part by adopting more comprehensive measures of spending and utilization than what are found in the most closely related literature. Specifically, our analysis examines usage across all facilities, providers, and care settings within a given time horizon (i.e., an "episode of care"). Each episode is initiated by a planned and elective inpatient procedure and encompasses all claims up to 30 days prior and 90 days after the inpatient stay. Each episode therefore includes all spending and utilization related to inpatient, outpatient, skilled nursing facility (SNF), home health, and professional services. On average, the initiating inpatient stay accounts for nearly $60 \%$ of total episode spending, which means that over $40 \%$ of episode spending is potentially malleable and affected by the physician's relationship with the hospital. Our episodes of care therefore incorporate mechanical increases in outpatient facility spending due to site-of-care payment differentials, as has been well-studied in the literature, but also changes in professional services and other care related to referral decisions once physicians transition from being practice owners to hospital employees.

To better understand the mechanisms through which hospital-physician vertical integration can impact these episode outcomes, we develop a theoretical framework. In our model, an episode of care is initiated by a physician/hospital pair, in consultation with the patient. The physician and hospital then maintain some influence on treatment patterns throughout the episode (e.g., follow-up visits, testing, or related consultations), even if care is not delivered at the same hospital or by the same physician. For example, the operating physician, as the focal provider in such a clinical context, would typically dictate the pre-operative care and post-operative discharge plan, guiding the patient on necessary follow-up care in order to achieve the targeted post-surgical health outcomes (e.g., better patient mobility and reduced pain following a joint replacement). The operating physician is also likely to advise the patient on the timing and frequency of follow-up care, either delivered by the operating physician herself or other providers.

Our main analysis is based on a $20 \%$ random sample of Medicare fee-for-service (FFS) beneficiaries from 2010 to 2015, for which we observe all Parts A and B Medicare claims. As discussed in more detail in Section 3, our final analytic dataset consists of 1,030,031 episodes, covering 67,235 
unique physicians and 4,128 unique hospitals. Data on hospital ownership of physician practices comes from the SK\&A physician survey database. These data offer several advantages compared to other common measures of alignment, such as a hospital's self-reported classification within the American Hospital Association (AHA) annual surveys (Cuellar \& Gertler, 2006; Ciliberto \& Dranove, 2006; Baker et al., 2014). For example, measures of integration at the hospital level cannot identify variation across physicians operating at the same hospital. Our unique combination of data can overcome this challenge and is therefore a clear improvement in this regard. Finally, we supplement the claims data with information on hospital characteristics and county demographics from a variety of sources, including the AHA annual surveys and the American Community Survey (ACS).

For each episode, our outcomes include total physician and facility Medicare spending and number of claims, as well as quality measures including mortality, readmissions, and complications. ${ }^{3}$ Our regression specifications include physician-hospital fixed effects as well as a rich set of patient, hospital, and market characteristics. The key variable of interest in all cases is an indicator for whether the operating physician is part of a practice owned by the hospital system.

Due to endogeneity concerns arising from the two-sided matching process by which physicians and hospitals become vertically integrated, we propose an instrumental variables (IV) estimate for vertical integration using a 2010 Medicare payment shock as our instrument. Dranove \& Ody (2019) examine this payment shock in detail, showing that the shock had a significant and meaningful effect on hospital acquisitions of physician practices. The purpose of the payment shock, as discussed in more detail in Section 4, was to update physician payments using new survey information. The update differentially affected services provided in an office setting versus a facility setting, thereby increasing the incentive for hospitals to acquire certain physician practices. We construct as our instrument the total additional revenue gained per physician practice (due to the payment updates) if the visits were conducted in a facility setting (or billed as such) compared to a physician's office.

From our IV analysis, we estimate an economically small and statistically insignificant change in episode-level Medicare payments following vertical integration, alongside a relatively large reduction in the intensity of services per episode. Specifically, our point estimates suggest a statistically insignificant $2 \%$ increase in Medicare payments per episode among vertically integrated physicianhospital pairs, with around 26 fewer claims per episode (a 35\% reduction on a base of 73 total claims per episode). ${ }^{4}$ When we decompose each episode into spending for inpatient, outpatient (facility),

\footnotetext{
${ }^{3}$ We focus on the relationship between vertical integration and spending/utilization; however, effects on quality are clearly relevant when interpreting any effects on spending. We therefore present results for quality in the supplemental appendix. In general, we find null effects of vertical integration on quality, consistent with findings in Carlin et al. (2015a) and Koch et al. (2018), among others.

${ }^{4}$ We focus much of our discussion on the IV estimates, with OLS estimates as well as event study estimates following Callaway \& Sant'Anna (2020) presented in the supplemental appendix. Our OLS and event study estimates align with
} 
SNF, home health, and professional services, we find relatively large increases in outpatient spending, coupled with large reductions in the quantity of professional services, consistent with effectively no change in episode spending overall. Our point estimates for outpatient spending generally align with Koch et al. (2017); however, our findings suggest that, in the context of elective surgical care, this increase is ultimately offset by a reduction in total number of professional claims when the surgeon is vertically integrated. We also find that the reduction in professional service claims derives entirely from a reduction in visits to non-integrated providers. As such, hospitals appear to keep more care within the system when owning the focal physician agent, which reveals an indirect channel for financial benefits from a given practice acquisition. Our results are consistent with newly integrated physicians conforming to the referral patterns of their peers that were previously integrated with the same hospital.

Given the potential for integration to disincentivize effort, we also examine the effects of vertical integration on physician effort, measured both on the intensive margin (number of claims and spending within an episode) and overall (total claims and spending in the year). We find little evidence that physicians actively reduce the amount of care per patient in the absence of direct financial incentives, conditional on an elective procedure taking place. Conversely, we find that physicians significantly decrease their total number of claims in a year, with a net decrease of nearly 600 claims on a base of nearly 2,200 per year. This decrease derives predominately from a reduction in professional services; however, the largest effects in terms of Medicare payments derive from a decrease in operations. Specifically, we estimate that integrated physicians initiate 4 fewer episodes per year, accounting for a loss of around $\$ 100,000$ in inpatient revenue to the acquiring hospital. These results are consistent with weaker incentives for own effort after hospital employment.

Our findings contribute to two related literatures. Our first contribution is to the broader literature on physician agency. In documenting physician agency problems, authors typically examine changes in physician treatment choices due to relative differences in financial incentives across services (e.g., Afendulis \& Kessler (2007), Clemens \& Gottlieb (2014), Gruber \& Owings (1996), and Iizuka (2012), among others). The presence of physician agency problems is also implicit in studies of unexplained geographic variation in health care utilization (e.g., Finkelstein et al. (2016) and Molitor (2018), among many others). Our analysis identifies physician-to-physician referrals as another important dimension of physician agency, whereby hospitals appear to use vertical integration to limit referrals to non-integrated physicians. In this way, our work highlights the physician's relationship with the hospital as a potential mediating factor in understanding effects of physician agency on patient care, which also aligns with several recent studies focused on diagnostic testing quantities and referral the IV results, albeit with smaller effect sizes. 
choices when physicians are hospital-owned (Chernew et al., 2021; Whaley et al., 2021; Young et al., 2021).

In addition, our work directly connects to the literature on the effects of vertical integration on various outcomes, such as physician practice prices (Capps et al., 2018), hospital prices (Baker et al., 2014; Lin et al., 2021), changes in location of professional services (Koch et al., 2017; Richards et al., 2022; Whaley et al., 2021), changes to physician referrals (Carlin et al., 2015b; Baker et al., 2016; Walden, 2016), total spending (?Baker et al., 2016; Capps et al., 2018), or quality of care (Koch et al., 2018; Carlin et al., 2015a). While it is clear from this literature that vertical integration affects physician and hospital behaviors (potentially in socially undesirable ways), some of these changes may offset each other. For example, higher-priced services may not translate to higher spending if integration also affects the quantities of care consumed. Likewise, more inward (or within-organization) referrals to complementary providers may blunt some competitive forces and/or forgo lower cost options, but it may also lead to less duplication of services and/or more effective management of the patient overall. Analyses of a given care attribute (e.g., service price) or care decision (e.g., site of care delivery) cannot adequately speak to these broader considerations, which leaves the overall effects of vertical integration on spending and utilization unclear.

Our paper extends this literature in several important ways. First, our analysis is intentionally broader and speaks to the overall effects of integration, accounting for dimensions by which spending may increase (e.g., replacing office-based visits with outpatient visits) while also considering potentially offsetting behaviors (e.g., a reduction in referrals to other physicians, labs, imaging, etc., or an overall reduction in physician effort). Our focus on episodes for elective surgical care reflects a second way in which we extend the existing literature. Such elective procedures are highly common to Medicare beneficiaries and are known to be vitally important for hospitals' revenues and profit margins. Moreover, elective surgeries have the benefit of lending themselves to clinically and analytically well-defined episodes (or "bundles") of care. Therefore, we believe that our careful construction of elective surgical care episodes and comprehensive assessment of quantities of care and spending within the episode represent a novel and much needed addition to the body of evidence around provider consolidation in U.S. health care.

Third and related, beyond our ability to capture the overall effects of health care utilization and spending, our clinical setting also provides an ideal context to explore disincentives to work (i.e., engage in billable activity) after transitioning from self-employment to a hospital employee-a margin that has not been studied in the literature, to the best of our knowledge. Surgeons are, by definition, proceduralists, with the bulk of their revenue generation directly connected to the provision of surgical interventions. We are also able to carefully quantify within-episode ancillary care provided inside 
versus outside the physician's new parent organization. In other words, we not only demonstrate if the physician's own billable effort changes following integration, but we also assess if she alters the intensity and source of ancillary care under her guidance and how this influences the overall cost and quality of care. Doing so speaks to the informal/indirect incentives emanating from the larger firm as well as potential efficiency gains when otherwise disconnected services are provided by physicians linked by common ownership and information technology.

Due to our focus on the overall effects of integration (rather than more isolated responses), the scale and scope of our data, our emphasis on physician effort, and our attention to endogeneity, our analysis offers a more complete picture of how vertical integration affects physician treatment patterns and health care utilization. From a policy perspective, our results using the full episode of care suggest smaller effects of vertical integration on spending that would be estimated when focusing specifically on the role of facility fees or on spending at the acquiring hospital. Shifting to site neutral payments may still generate public insurer-and hence taxpayer-savings for one-off procedures, but when considering a bundle of surgical care, the simultaneous movements in reimbursement rates and quantities of care matter. That said, under a site-neutral payment regime, Medicare would have experienced savings through lower total episode payments overall in our care delivery context. The other important margins along which integration may have meaningful effects are prices ( which is irrelevant in our setting of Medicare fee-for-service) and quality of care, each of which have also been well-studied in the literature (and the latter of which we verify in our data as well). Specifically related to antitrust oversight of vertical integration, our results also suggest that concerns regarding increases in Medicare spending and health care utilization due to integration are secondary to concerns regarding price increases among commercial insurers. However, vertical integration could put nonintegrated physician practices at a disadvantage due to reduced referrals. Its long-run competitiveness effect awaits future research.

\section{Motivation}

We motivate our empirical analysis with a simple model of physician behavior (Ellis \& McGuire, 1996; Finkelstein \& McGarry, 2006). We assume physician $j$ and hospital $k$ initiate an episode of care for patient $i$, the goal of which is to maximize the perceived utility of patient $i$ as well as the profits of the physician (or the physician's practice) or the hospital. The total amount of care delivered in this episode is denoted $y_{i j k} .{ }^{5}$ Note that the initiating physician-hospital pair is not assumed to directly

\footnotetext{
${ }^{5}$ To simplify notation, we let the subscript $j$ denote physician $j$ within a given practice. Our empirical analysis ultimately restricts the sample to physicians that operate in a single practice, so that we can uniquely assign practice
} 
provide all care in the episode - they need only maintain some influence on treatment patterns throughout the episode.

Given the inherent differences in revenue generation for a physician-owned versus hospital-owned practice, we split this optimization problem between physicians that are vertically integrated with a hospital (VI) and those that are non-integrated (NI). ${ }^{6}$ Denote profits to a non-integrated physician by $\pi_{j, N I}=R_{j}\left(y_{i j k}\right)-c_{j, N I}\left(y_{i j k}\right)$, where $R_{j}$ captures the net revenue to the physician (reimbursement net direct costs of patient care) and $c_{j, N I}$ denotes other indirect costs not reimbursed by insurers. Denote profits to the hospital by $\pi_{k}=R_{k}\left(y_{i j k}\right)-c_{k}\left(y_{i j k}\right)$ and perceived patient utility by $\tilde{u}\left(y_{i j k}\right)$. Omitting subscripts on the revenue and cost functions, we assume that $R^{\prime}\left(y_{i j k}\right)>0$ and $R^{\prime \prime}\left(y_{i j k}\right) \leq$ $0 ; c^{\prime}\left(y_{i j k}\right)>0$ and $c^{\prime \prime}\left(y_{i j k}\right)>0$; and $u^{\prime}\left(y_{i j k}\right)>0$ and $u^{\prime \prime}\left(y_{i j k}\right) \leq 0$. Assuming that the physician's objective is additively separable in perceived patient utility and profits, the non-integrated physician's objective function is

$$
y_{i j k}^{N I}=\arg \max _{y}\left\{\theta_{u} \tilde{u}\left(y ; \Gamma_{k}, \Gamma_{j}, \kappa_{i}\right)+\theta_{\pi}^{j} \pi_{j, N I}\left(y ; \Gamma_{k}, \Gamma_{j}, \kappa_{i}\right)\right\}
$$

where $\Gamma_{k}, \Gamma_{j}$, and $\kappa_{i}$ reflect hospital, physician, and patient characteristics, respectively, and $\theta_{u}$ and $\theta_{\pi}^{j}$ capture different weights that the physician places on perceived patient utility and own-profits. The solution to the non-integrated physician's optimization problem therefore satisfies the following,

$$
\theta_{u} \tilde{u}^{\prime}\left(y_{i j k}^{N I}\right)=\theta_{\pi}^{j}\left(c_{j, N I}^{\prime}\left(y_{i j k}^{N I}\right)-R_{j}^{\prime}\left(y_{i j k}^{N I}\right)\right)
$$

Similarly, denote the profits to a vertically integrated physician by $\pi_{j, V I}=\bar{R}-c_{j, V I}\left(y_{i j k}\right)$, where $\bar{R}$ denotes a fixed salary received from the acquiring hospital. The vertically integrated physician's objective function is

$$
y_{i j k}^{V I}=\arg \max _{y}\left\{\theta_{u} \tilde{u}\left(y ; \Gamma_{k}, \Gamma_{j}, \kappa_{i}\right)+\theta_{\pi}^{j} \pi_{j, V I}\left(y ; \Gamma_{k}, \Gamma_{j}, \kappa_{i}\right)+\theta_{\pi}^{k} \pi_{k}\left(y ; \Gamma_{k}, \Gamma_{j}, \kappa_{i}\right)\right\}
$$

reflecting the fact that a vertically integrated physician may incorporate hospital profits into their objective function. The weight assigned to the hospital's profit function in the physician's optimization problem is captured by $\theta_{\pi}^{k}$. The optimal amount of care (from the physician's perspective) for an characteristics (including ownership by a hospital system) to the physician.

${ }^{6}$ The type of integration in this paper, that of a hospital purchasing a physician practice, differs from some recent studies in the literature. Starc \& Town (2020), for example, examines the effects of integration in the design of Medicare Advantage benefits, while Cuesta et al. (2019) considers the effects of vertical integration between hospitals and insurers. 
integrated physician is then reflected by

$$
\theta_{u} \tilde{u}^{\prime}\left(y_{i j k}^{V I}\right)=\theta_{\pi}^{j} c_{j, V I}^{\prime}\left(y_{i j k}^{V I}\right)+\theta_{\pi}^{k}\left(c_{k}^{\prime}\left(y_{i j k}^{V I}\right)-R_{k}^{\prime}\left(y_{i j k}^{V I}\right)\right)
$$

Comparing Equations 1 and 2, and assuming that perceived patient preferences remain unchanged, we see that the optimal amount of care for a vertically integrated versus non-integrated physician depends largely on two factors. First, as an employee, an integrated physician's marginal revenue for own-profits drops to zero. All else equal, this would tend to reduce the amount of care in an episode. This is a classic agency mechanism in which salaried arrangements disincentivize effort relative to being self-employed. We consider these effects in Section 8, where we examine physician effort both on the intensive margin (i.e., conditional on an elective procedure taking place) and overall (i.e., total physician claims and spending per year). Within an episode, note that the operating physician accounts for around $20 \%$ of all physician office visits in an average episode, suggesting an opportunity for economically meaningful reductions in physician's own-effort within an episode. Nonetheless, we suspect that effects of integration on physician's own-effort manifest more at the extensive margin (e.g., accepting more or fewer patients throughout the year). Our empirical analysis therefore also considers more aggregate measures of physician effort (i.e., total billable activity) using total physician-level spending and claims in a year. These analyses would allow us to capture the overall effect of integration on physician effort.

Second, for a vertically integrated physician, the hospital's profit function is now part of their optimization problem. The extent to which this affects treatment decisions depends on the relative size of $\theta_{\pi}^{k}$ versus $\theta_{\pi}^{j}$. If $\theta_{\pi}^{k}$ is sufficiently large, then we would expect an adjustment to treatment patterns that is more financially favorable to hospitals. ${ }^{7}$ The introduction of the hospital's profit function into the physician's optimization problem therefore introduces the potential for hospitals to influence physician behaviors toward the hospital's financial gain.

There are two primary ways in which we envision a hospital influencing physician treatment decisions in our setting. ${ }^{8}$ First, hospitals may influence where care is delivered. For example, a physician can choose to operate in the acquiring hospital, $k$, as opposed to some other hospital for which the physician also has admitting privileges. Vertical integration is then a mechanism by which the hospital increases the probability of the physician operating in their hospital relative to that of a

\footnotetext{
${ }^{7}$ It is reasonable to assume that the physician's marginal costs do not meaningfully change after integration with a hospital; however, integration may reduce practice costs by lowering the administrative burden of seeing patients, or integration may increase the costs of communicating with non-integrated physicians (e.g., due to barriers in sharing information via electronic medical records). Such costs are notoriously difficult to measure, and we have no such measures available in our data.

${ }^{8}$ Although not part of our analysis, researchers also posit that vertical integration may facilitate some form of product bundling wherein hospitals group many services together (Madison, 2004; Afendulis \& Kessler, 2007).
} 
competitor hospital (Baker et al., 2016). Moreover, integrated physicians may bill for the same visit as an outpatient service instead of a standard office visit, taking advantage of the fee differentials (a motivation for increased physician-hospital integration) even though the nature and physical location of the visit itself is identical. Koch et al. (2017) find strong empirical evidence of such behavior. This dimension of hospital influence will tend to increase total spending but may not effect the quantity of care.

Second, hospital influence may determine which providers a patient visits throughout the episode. Specifically, hospitals may prefer that patients only visit other physicians within the same hospital system, otherwise restricting visits to non-integrated physicians. Imposing these forms of restrictions may confer additional bargaining power to the hospital (Gowrisankaran et al., 2015; Ho \& Lee, 2017) and lead to higher physician and hospital prices for commercially insured population (Capps et al., 2018; Baker et al., 2014; Lin et al., 2021). Of particular interest to our setting, this dimension of hospital influence will tend to increase the amount of care to other integrated physicians (since such visits contribute directly to the hospital's revenue) at the expense of non-integrated physicians. Whether the total amount of care and spending will decrease or increase remains an empirical question.

Ultimately, this discussion suggests that vertical integration creates both upward and downward pressure on the total amount of care, with potentially separate effects on spending. Downward pressure derives from the loss of marginal revenue from the physician's profit function, and potentially from a reduction in care delivered by other, non-integrated physicians, while upward pressure derives from the standard financial incentive to provide more care, all else equal. Fully examining the effects of vertical integration on the amount of care therefore requires a more comprehensive measure of treatment, as reflected in our episode-based outcomes.

Empirically, we assume $\tilde{u}, \pi_{j}$, and $\pi_{k}$ are additively separable in $i$ and $(j, k, t)$. A reduced-form analog to Equations 1 and 2 then follows:

$$
y_{i j k t}=x_{i t} \beta_{x}+w_{j k t} \beta_{w}+\mathrm{VI}_{j k t} \delta+\gamma_{j k}+\lambda_{t}+\epsilon_{i j k t},
$$

where $x_{i t}$ denotes a vector of patient characteristics, some of which may be time-invariant; $w_{j k t}$ denotes observed and potentially time-varying hospital and county characteristics; $\mathrm{VI}_{j k t}$ denotes an indicator for whether hospital $k$ owns the practice of physician $j$ at time $t ; \gamma_{j k}$ denotes time-invariant physician-hospital fixed effects; $\lambda_{t}$ denotes time fixed effects; and $\epsilon_{i j k t}$ is an error term. The key variable of interest is the indicator for whether the physician practice is owned by the hospital, $\mathrm{VI}_{j k t}$. We consider this overall effect in Section 5. We then attempt to decompose this overall effect into the two areas discussed above. First, we examine changes due to reallocation of care, which we argue is 
a reflection of heterogeneities in treatment effects within an episode. This is the subject of Section 6. Second, we consider effects of vertical integration on visits to different types of physicians. This analysis is in Section 7. As part of this analysis, we also consider the effects of vertical integration on visits to the same physician, which we take as a proxy for the effect of vertical integration on the physician's own-effort on the intensive margin (measured in billable visits per episode). We then extend this analysis to a measure of total physician-level spending and claims per year in Section 8 .

\section{Data}

\subsection{Dataset Construction}

Our analysis aims to quantify the overall effects of vertical integration by examining spending and utilization over an extended, well-defined period rather than a single visit. We refer to this extended period as an "episode of care," adopting the language from CMS regarding bundled payments. We define an episode as all care observed during a 90-day period after a planned inpatient stay as well as all professional services (i.e., "carrier" claims) incurred 30 days prior. ${ }^{9}$ We define a planned admission as an elective admission type that is initiated by a physician or clinic, as captured in the admission source variable in the Medicare claims data. This excludes, for example, transfers from other hospitals or inpatient stays initiated through the emergency department, urgent care center, or trauma center. Our analysis is based on Parts A and B claims for a 20\% sample of Medicare beneficiaries from 2010 through 2015. We also employ data from 2009 in constructing our instrument, but we focus on the 2010-2015 period for our analysis as those are the years in which our instrument offers identifying variation.

Planned procedures are of interest as they facilitate clinically and analytically well-defined episodes (or "bundles") of care. This is not typically the case for many genres of physician services, including primary care; however, in the context of planned inpatient surgeries, examining episodes of care is both feasible and advantageous as it allows a comprehensive assessment of all care delivery happening within the demarcated episode-knowing that the focal surgeon and hospital exercise considerable influence on the resulting quantities of care, where care takes place, and who delivers these ancillary services. As a sensitivity analysis, we also limit our sample only to major orthopedic procedures, with similar overall findings. Those results are included as part of our specification curves discussed

\footnotetext{
${ }^{9}$ This definition and construction of an episode follows recent guidance from CMS in calculating bundled payments for joint replacement (Mechanic, 2015). Ellimoottil et al. (2017) also finds that this broad construction of an episode highly correlates with a more clinically narrow definition, such as the episodes constructed in the hospital compare data and the RAND health insurance experiment (Aron-Dine et al., 2013).
} 
in Section 5.

We construct a dataset of all 90-day episodes from 2010 through 2015, where each episode is initiated by a planned and elective inpatient stay. All episodes in our final data are non-overlapping, so that any elective inpatient stay observed within an existing episode is treated as an inpatient stay in that episode rather than as initiating a new episode. The operating physician associated with the initial inpatient stay is assigned as the physician to that episode. Similarly, the admitting hospital for the initial inpatient stay is assigned as the hospital for the episode. We restrict the sample to individuals aged 65 and above, and we drop episodes in which Medicare payments fall in the highest or lowest one-percent of all observed amounts in a given year. Our final dataset consists of a total of 1,030,031 episodes, where we observe 90,799 unique physician/hospital pairs, based on 4,128 unique hospital NPIs and 67,235 unique physician NPIs.

We incorporate additional hospital-level data from the provider of service (POS) files and the AHA annual surveys, which we merge to the episode data using the Medicare provider number. These data include the number of staffed hospital beds and indicators for hospital teaching status, membership in a larger hospital system, and for-profit/not-for-profit ownership. We then incorporate hospital financial information and data on total discharges from the Healthcare Cost Report Information System (HCRIS), again merged based on the Medicare provider number. Finally, we incorporate local demographic and other county-level variables from the American Community Survey (ACS), merged based on county FIPS codes observed in the AHA data.

We then merge data on hospital ownership of physician practices from SK\&A, a commercial research firm that regularly surveys the ambulatory physician practice landscape. The SK\&A database approximates a near-universe of U.S. office-based physician practices and provides detailed information regarding practice ownership affiliations (including the health system name for those vertically integrated), practice specialty, practice size, and practice location. ${ }^{10}$ The SK\&A data also includes each physician's NPI, which we use to merge to the claims data.

\subsection{Dependent Variables}

Our unit of observation is a 90-day episode beginning with a planned and elective inpatient stay, and our main outcomes are Medicare payments and number of claims per episode. These totals include all Parts A and B claims except for durable medical equipment (DME) and hospice care. Our outcomes therefore capture the overwhelming majority of all health care utilization within each episode and over $75 \%$ of annual spending per patient. We also consider several measures of quality, including 90-

\footnotetext{
${ }^{10}$ Since SK\&A focuses on the office-based physician practice landscape, our analysis does not consider hospitalists or other forms of hospital-based physician employment.
} 
day mortality, 90-day readmissions, and other complications. We measure complications based on the incidence of sepsis and surgical site infections (SSI) within the 90-day episode. All quality measures are therefore constructed based on the same 90-day time frame as our spending measures. We define "any" complication as whether the patient is diagnosed with sepsis or SSI over the episode. Lists of ICD-9 diagnosis codes used to identify sepsis and SSI are included in the supplemental appendix. Since quality is of secondary interest in this analysis, we present our results on vertical integration and quality in the supplemental appendix. Across all specifications and estimators, we find no evidence that vertical integration meaningfully improves quality, consistent with previous findings such as Koch et al. (2018).

\subsection{Independent Variables}

Our primary independent variable of interest is an indicator for whether physician practice $j$ is owned by hospital $k$ (or the system to which hospital $k$ is a member), which is captured within $z_{j k t}$ in Equation 3. Our "treatment" is therefore defined at the physician-hospital level, with the operating physician and admitting hospital serving as the treated pair, consistent with the expositional framework in Section 2. This is not to suggest that the operating physician or admitting hospital has full control over all episode payments, but that they can have a large influence on treatment patterns before and after discharge. Intuitively, we consider vertical integration as one way in which hospitals can gain further control over pre- and post-discharge treatment patterns, and we assume that any such control is exercised in concert with the operating physician.

We also include in $z_{j k t}$ the following observables: 1) hospital characteristics, including the number of full-time equivalent (FTE) nurses, the number of FTE other medical staff, and indicators for whether the hospital is part of a larger system, for-profit versus not-for-profit ownership, and designation as a major teaching hospital; ${ }^{11}$ and 2) county characteristics, including a set of variables that capture the county's distribution of age, income, gender, education, and race, as well as indicators for whether the county hospital market is a monopoly, duopoly, or triopoly.

Since the same patient will not (on average) have several episodes for a given physician-hospital pair, we do not include patient-specific fixed effects. We instead include in $x_{i t}$ a set of variables intended to capture the total health care utilization for patient $i$ in years prior to time $t$. Specifically, we sum all claims and Medicare payments for each patient in all years before $t$, calculate (separately) the quartiles for each of these two variables, and form six indicator variables for whether the patient

\footnotetext{
${ }^{11}$ The AHA survey data includes measures of physician and resident FTEs; however, we exclude such characteristics from our set of hospital-level controls since the acquisition of a physician practice will tend to also affect the number of physician or resident FTEs at the hospital.
} 
falls in the $2 \mathrm{nd}$, 3rd, or 4th quartile of each variable. ${ }^{12}$ We also include in $x_{i t}$ a dummy variable for the patient's race, sex, and set of dummy variables for the DRG code, all based on the initiating inpatient stay for each episode.

\subsection{Descriptive Statistics}

Descriptive statistics for the episode-level data are provided in Table 1, as well as histogram of the 10 most frequently occurring DRGs in Figure 1. Consistent with national trends in inpatient care, we see a reduction in the number of episodes per year from over 192,000 in 2010 down to 167,000 in $2014 .{ }^{13}$ We see also from Table 1 that an average 90-day episode incurs around \$25,000 in Medicare payments and over 70 separate claims. We also present summary statistics for our selected quality measures. On average, mortality rates per episode are $3.5 \%$, with readmission rates of $16.4 \%$ and complication rates (defined as "any" complication) of 3.5\%. Finally, Table 1 shows that $37 \%$ of episodes are initiated by an integrated physician-hospital pair in 2015 compared to nearly $12 \%$ in 2010.

Tables 2-3 present descriptive statistics for individual physicians and hospitals, respectively. From Table 2, our final data consist of around 5 episodes per physician per year. ${ }^{14}$ Also from Table 2, we see that around $13 \%$ of physicians in our data were integrated with a hospital or hospital system in 2010 compared to nearly $40 \%$ in 2015 . Recall that integration is defined at the hospital system and practice level, so that physicians can operate at multiple hospitals within the same system that owns their practice. Physicians may also (and do) operate at hospitals outside of the system that owns their practice, such that the percentage of episodes initiated by an integrated physician-hospital pair in Table 1 should be slightly lower than the percentage of integrated physicians in Table 2.

Table 3 describes the average hospital in our data. From Table 3, there are about 51 episodes per year in a given hospital, ${ }^{15}$ and the initiating hospitals have an average of 218 staffed beds. Around 16\% of hospitals in our data are designated as for-profit, which is lower than overall rates in the AHA data, and the percentage of hospitals reporting membership in a larger hospital system increased slightly from $60 \%$ in 2010 to $69.2 \%$ in 2015 . Our analytic sample therefore skews more toward larger, not-for-

\footnotetext{
${ }^{12}$ In calculating total Medicare payments or claims, we make no restrictions on the type of procedures and include all observed claims for each patient. This reflects substantially more observations per patient because the observations are not restricted to be planned and elective procedures.

${ }^{13}$ Note that this decrease in inpatient care is not driven by shifting to outpatient care since most of the procedures captured in our analysis could not be done in an outpatient setting. We restrict our 2015 data to January-September in order to ensure that we observe all claims for 90 days after the inpatient stay.

${ }^{14}$ In our specification curves in Figure 5, we include estimates when limiting the sample to physician-hospital pairs with more episodes, with little change in the results.

${ }^{15}$ Adjusting for our $20 \%$ sample, our data reflect around 250 episodes per hospital per year. This count of episodes is broadly consistent with other studies in the literature. For example, Dummit et al. (2016) report around 200 "lower extremity joint replacement admissions" per year in their study of the hospital bundled payment program. We include a broader set of conditions but with the restriction to planned and elective inpatient stays as per the admission source codes.
} 
profit systems. Finally, the percentage of hospitals that are identified as owning at least one physician practice (or hospitals affiliated with a larger system that owns a practice) almost doubled from nearly $38 \%$ in 2010 to over $65 \%$ in 2015.

\section{Estimation Strategy}

Our primary independent variable of interest is an indicator for whether physician $j$ 's practice is owned by hospital $k$ or the larger system to which hospital $k$ is a member. To estimate this effect, we first present the results of a difference-in-differences estimation based on the estimator proposed in Callaway \& Sant' Anna (2020). ${ }^{16}$ Here, we define the treated group as any physician-hospital pair that becomes vertically integrated in a given year, and our control group consists of all physician-hospital pairs that are not vertically integrated at any point over our time period. For this analysis, we exclude all control variables in order to focus solely on the change in episode-level outcomes before/after integration.

The results are presented in Figure 2, where the top panel presents event study results for log payments, and the bottom panel presents results for the number of claims. Figure 2 reveals a strong negative relationship between integration and claims, with a slight increase in Medicare payments per episode; however, there are at least two major barriers to assigning a causal interpretation to such an analysis: 1) patient sorting; and 2) provider sorting.

Regarding patient sorting, patients may prefer vertically integrated physician-hospital pairs over non-integrated pairs for unobserved reasons that are correlated with health care utilization. In addition, a patient's decision to undergo inpatient surgery might be affected by physician-hospital integration. To address these concerns, we first argue that in our setting, patient selection of getting an inpatient surgery constitutes a relatively minor source of endogenity. This is because we examine episodes initiated with a planned and elective inpatient stay, the overwhelming majority of which are joint replacements or reattachment of lower extremity without major complications. During our study period, those procedures are not allowed in an outpatient setting. It is therefore very uncommon that integration would affect the decision to undergo inpatient procedure. In addition, we attempt alleviate the potential bias due to patient sorting (in particular, patient sorting into physician-hospital pairs) by utilizing a very rich set of controls, including measures of the patient's entire history of Medicare claims and spending, DRG codes for the initiating inpatient say, and a set of physician-hospital fixed effects.

Regarding provider sorting, hospitals may actively pursue the purchase of physician practices

\footnotetext{
${ }^{16}$ We also present ordinary least squares (OLS) estimates of Equation 3 in the supplemental appendix.
} 
for unobserved reasons that are also related to physician treatment patterns. Similarly, physicians may actively pursue being acquired by certain types of hospitals, again for unobserved reasons that are correlated with treatment patterns. To address this concern, we pursue an instrumental variables (IV) estimator that exploits a plausibly exogenous update to the physician fee schedule in 2010 as an instrument. Dranove \& Ody (2019) study this price shock in detail and present strong empirical evidence that the payment change affected physician-hospital vertical integration; however, since our analysis focuses on a different set of physicians in a different empirical context, we explain and revisit some of their analysis here before presenting the IV results.

\subsection{Institutional Background}

To better understand the update to the physician fee schedule, recall that CMS pays physicians an administratively set fee based on the relative value units (RVUs) of each service. There are three RVUs for any given procedure or service: 1) a work RVU, which is an estimate of the cost of the physician's work for each service; 2) a malpractice RVU, which is an estimate of the malpractice costs for each service; and 3) a practice expense RVU, which is an estimate of the practice expenses for each service and may differ depending on the location of the service (e.g., a physician's office versus outpatient facility). The practice expense RVU is further split into direct and indirect expenses, where the indirect expenses are calculated as some percentage of direct expenses. CMS then takes the sum of these three RVUs and multiplies by an RVU-to-dollar conversion factor in order to find the final dollar figure for each service.

Prior to 2010, CMS relied in-part on the American Medical Association's (AMA's) Socioeconomic Monitoring System survey to calculate the indirect/direct cost ratios in the practice expense RVUs. This information was eventually perceived as outdated, and in 2007-2008, CMS deployed a new survey to update their indirect/direct cost estimates. This new survey was the Physician Practice Information Survey (PPIS). As highlighted in Dranove \& Ody (2019), the PPIS acts as a shock to the payment differential between services provided in an office versus facility setting. Acquiring physician practices, and thus exploiting the payment differentials between office and facility settings, became more or less attractive to hospitals due to the PPIS. Note that the PPIS price shock was phased in over a four-year period, with 1/4th of the full price differential introduced in 2010,1/2 of the differential in 2011, 3/4ths of the differential in 2012, and the full payment differential in 2013. In our following analysis, we allow for the price shock to have differential effects over the years. 


\subsection{Calculation of PPIS Price Changes}

Following Dranove \& Ody (2019), we measure the price changes due to PPIS in two steps. First, we construct the price change relative to the baseline 2009 price, separately for facility and non-facility (e.g., office-based) payments and separately for each HCPCS code, $c$. We denote these price changes by $\Delta p_{f, t}^{c}=p_{f, t}^{c}-p_{f, 2009}^{c}$ for facility payments and $\Delta p_{n f, t}^{c}=p_{n f, t}^{c}-p_{n f, 2009}^{c}$ for non-facility payments. Second, we construct the relative price differential, $\Delta p_{r, t}^{c}=\Delta p_{f, t}^{c}-\Delta p_{n f, t}^{c}$, as the difference in price changes between facility and non-facility payments. This reflects the additional distortion in facility versus non-facility payments introduced by the PPIS.

We then aggregate $\Delta p_{r, t}^{c}$ up to the physician level based on the physician's count of office-based claims for HCPCS code $c$. We use 2008 claims for physician $j$ in order to avoid additional endogeneity of claim counts due to the price changes, denoted by $q_{j(c), 2008}$, and we weight this measure by 2008 non-facility prices. Finally, we normalize this total revenue change by the non-facility revenue in 2008. The resulting physician-level measure is:

$$
\Delta \text { Revenue }_{j, t}=\frac{\sum_{c} \Delta p_{r, t}^{c} \times q_{j(c), 2008} \times p_{n f, 2008}^{c}}{\sum_{c} q_{j(c), 2008} \times p_{n f, 2008}^{c}} .
$$

In constructing the quantities, $q_{j(c)}$, we exclude all HCPCS codes that appear to be "exclusive" to a non-facility setting, which we define as occurring over $90 \%$ of the time in a non-facility setting versus a facility setting. For consistency with Dranove \& Ody (2019), we also calculate an analogous measure of the relative revenue increase due to a different change to the physician fee schedule phased in from 2007 to 2010. We refer to the revenue change from the 2007 price shock as $\Delta$ Revenue $_{j, t}^{2007}$ and from the 2010 price shock as $\Delta$ Revenue $_{j, t}^{2010}$, noting that the revenue change from the 2007 price shock is calculated relative to a 2006 baseline price while the 2010 price shock is calculated relative to a 2009 baseline price.

\subsection{Evaluating the PPIS as an Instrument}

Since hospitals ultimately acquire physician practices rather than individual physicians, we construct our instrument as the total $\Delta$ Revenue $_{j, t}^{2010}$ at the practice level, based on physician tax IDs in 2009 (the year prior to our main analysis). Figure 3 presents the practice-level $\Delta$ Revenue $_{j, t}^{2010}$ over time, separately for practices that are vertically integrated at some point over our panel versus physicians that are never integrated. We see from the figure a clear diversion in the relative revenue gained from PPIS among vertically integrated and non-integrated physicians, which suggests that hospitals focused their acquisitions on physician practices with higher potential for revenue gains due to location-based 
payment differentials.

To examine this incentive more formally, we again follow Dranove \& Ody (2019) and estimate a linear probability model of vertical integration as a function of $\Delta \operatorname{Revenue}_{j, t}^{2010}$ and $\Delta \operatorname{Revenue}_{j, t}^{2007}$, interacted with year dummies. In Figure 4, we present the point estimates and 95\% confidence intervals for the coefficients on $\Delta$ Revenue $_{j, t}^{2010}$ and year interactions. The results show an economically large and statistically significant increase in the probability of integration from the PPIS price shock.

Note that our empirical application examines the effects of vertical integration on episode spending and utilization for elective inpatient stays. Such inpatient stays are not directly affected by our instrument since the set of procedures in our data generally cannot be provided in an office setting. Our identification exploits differentials in revenue changes for a physician practice between facility and non-facility payments. In our setting, the exclusion restriction is that our instrument only affects spending via its effect on vertical integration, conditional on other observables in the model as well as physician-hospital and time fixed effects. The exclusion restriction is plausible because hospitals cannot exploit such revenue differentials without acquiring the practice. This feature, combined with the previous discussion and results in Figures 3 and 4, offers compelling support for the use of the 2010 PPIS price shock as a plausibly exogenous source of variation in physician-hospital vertical integration.

\section{Overall Effect of Vertical Integration on Episode Care}

We employ the practice-level PPIS price shock as an instrument for observed integration. The firststage results, presented in more detail in the supplemental appendix, reveal a positive effect of the price shock on integration with a first-stage F-statistic of 300 on the price shock coefficient. We present the IV estimates in Table 4, with results for log payments in column 1 and number of claims in column 2. We estimate an economically small and statistically insignificant increase in Medicare spending. We also estimate a large reduction of nearly 26 claims per episode (35\%) following vertical integration. Collectively, these results suggest no increase in spending per episode alongside a large reduction in the number of claims per episode. This pattern of results is consistent with an increase in spending per claim (due to a movement from office-based physician visits to outpatient facilities) which is offset by a reduction the total number of claims. We investigate this pattern in more detail in Section 6.

We also present a specification curve in Figure 5 that shows the results of several alternative specifications and samples. First, note that our main specification underlying Table 4 includes an indicator for integration of the physician-hospital pair as well as an indicator for whether the hospital 
owns any physician practice. We include the latter term because non-integrated physicians continue to operate at hospitals that have acquired other practices, and similarly, physicians integrated with a given hospital may still operate in other hospitals in the same time period. The hospital-level measure of integration is nonetheless highly correlated with integration specifically for a given physicianhospital pair. We therefore re-estimate results when excluding the hospital-level vertical integration measure, with estimates reflected in the specification labeled as "Base" in Figure 5.

Second, we consider the role of hospital quality by including mortality as an additional covariatethe intuition being that vertical integration may be correlated with quality, which is also correlated with our utilization measures. One concern with this specification is that quality itself may be an outcome of vertical integration, so it is not clear that mortality should be included as covariate. Nonetheless, for completeness, we re-estimate our main specification when including mortality as a covariate, as reflected in the specification labeled as "Quality" in Figure 5.

Third, we consider several alternative sample restrictions. Our concern is that, while we restrict the analysis to episodes initiated by a planned, elective inpatient stay, there remains a wide variety of different procedures and thus physician types in our final dataset. In our alternative samples, we consider restrictions on the number of episodes per physician-hospital pair, thereby focusing on relatively more "familiar" physician-hospital pairs (i.e., excluding physician-hospital pairs with very few interactions over time). We also consider explicit restrictions on the types of surgeries, where we focus only on orthopedic conditions (spinal fusion and joint replacements) with DRGs 460, 461, 462, 469, and 470. The DRG restrictions are reflected in the specification curve with the "DRGs" label. Finally, as an additional form of risk adjustment, we restrict the sample to only patients that are discharged to home in the initial inpatient stay, as reflected by the label "Home" in the specification curve.

We estimate effects separately across all combinations of specifications and sample restrictions. The top panel of Figure 5 presents our specification curve for episode payments, and the bottom panel presents the specification curve for number of claims in the episode. In both cases, the highlighted dot denotes our estimates from the main specification in Table 4 (i.e., the full sample and the "Hospital VI" specification). This figure offers some reassurance that the findings from our preferred specification are robust to a range of specification/sample restriction combinations.

\section{Reallocation of Care across Treatment Settings}

Our estimated effects of vertical integration on Medicare payments in Table 4 and Figure 5 may appear small relative to other studies. For example, Koch et al. (2017) estimate an increase in outpatient 
expenditures of at least $18 \%$ among vertically integrated physicians, with some estimates as high as $50 \%$. However, one main distinction of our study is the construction of an episode-based total spending measure, which enables us to estimate the overall effect of integration on total patient spending. As highlighted in Section 2, physician-hospital integration could lead to a reallocation of treatment to the acquiring hospital and a substitution of office visits with outpatient centers, which tend to increase spending to the integrated hospitals and physicians. This is actually the main focus and finding of Koch et al. (2017). However, it is not clear how such reallocation of care would change the total amount of care and spending for a given episode. For example, the increased care flowing to the integrated hospital could be fully offset by a reduction in care outside of the integrated hospital, leading to no change in overall spending. Indeed, our results suggest that the overall effect on total spending is negligible, although we do see a large reduction in the total amount of claims.

Reallocation of care would tend to manifest as heterogeneous effects of vertical integration both within and across episodes. Within an episode, there may be increases in outpatient spending but decreases in other professional services. Across episodes, we anticipate the greatest opportunity for offsetting behavior is among otherwise high-utilization episodes. We consider each of these dimensions of heterogeneity throughout the remainder of this section.

\subsection{Heterogeneity within Episodes}

We split our episodes into five components: 1) inpatient claims (after the initial procedure); 2) institutional outpatient claims; 3) professional services claims; 4) SNF claims; and 5) home health care claims. For each component, we re-estimate Equation 3 using IV as explained in Section 4. Results are summarized in Table 5, where we present results both for payments and the count of claims. Note that we consider spending in levels, rather than logs, so that we can compare magnitudes across different outcomes. We also present event study estimates for each component in the supplemental appendix. As was the case with our overall outcomes in Figure 2, the event studies are qualitatively similar to our IV estimates in Table 5 but with smaller effect sizes.

Consistent with Koch et al. (2017), we estimate relatively large and statistically significant increases in outpatient spending following vertical integration. Specifically, our estimate on outpatient spending suggests an increase of over $\$ 300$ in outpatient spending per epsidoe among vertically integrated physician-hospital pairs, but we estimate a similarly large reduction of \$338 in spending on professional services. Our results for number of claims mirror those for spending. We also estimate a large increase of around $\$ 130$ on home health care and a comparable reduction in SNF spending, although the latter effect is imprecisely estimated. We conclude from Table 5 that the increase in 
institutional outpatient payments is largely offset by a decrease in spending on professional services.

We emphasize that our estimated reduction in payments for professional services is not a simple byproduct of splitting a physician office visit into a separate physician fee payment and outpatient payment after integration. ${ }^{17}$ This is because we also estimate a reduction in professional services claims overall, not just the amount paid. In other words, while integration will tend to reduce the Medicare payment under the physician fee schedule, the splitting of payments into an outpatient and physician component following integration would not (by itself) change the count of professional services claims. These results suggest that integration not only shifts office visits to an outpatient setting within the integrated system, but also affects care allocation across integrated and non-integrated providers.

\subsection{Heterogeneity across Episodes}

In this section, we examine potential heterogeneities in estimated effects along the distribution of our outcomes of interest. ${ }^{18}$ Specifically, we estimate unconditional quantile regressions using the same empirical specification as in Equation 3 (Firpo et al., 2009; Borgen, 2016). We present results in Figure 6, with results for Medicare spending in the top panel and claims in the bottom panel. Each panel in Figure 6 shows the point estimate and 95\% confidence interval of physician-hospital integration on the outcome of interest at the relevant quantile.

The top panel of Figure 6 shows little variation in estimated effects on Medicare spending. Conversely, the bottom panel shows that the largest effects on number of claims arise for episodes in the highest claim quantiles. This pattern of results is again consistent with offsetting behaviors within an episode, where the largest opportunity for reduction in claims would come from otherwise highutilization episodes. Note that our quantile regressions do not employ an instrument, complicating any comparisons between estimates in Figure 6 and our IV results in Table 4. We therefore focus on the pattern of results across quantiles rather than the magnitudes.

\footnotetext{
${ }^{17}$ As described in more detail in Dranove \& Ody (2019), "When an independent physician performs a procedure in a facility or a VI physician...performs a procedure, Medicare reduces the [physician fee schedule] PFS price and the facility submits a separate bill under Medicare's Outpatient Prospective Payment System (OPPS). Thus, Medicare pays a single fee for procedures performed in non-facilities and two separate fees for procedures performed in facilities or for procedures performed by VI physicians. In general, the latter, combined price is higher than the former, single PFS price."

${ }^{18}$ Since the time frame of our analysis slightly overlaps with the adoption of Medicare bundled payments, and since the bundled payment program largely affects orthopedic procedures, hospital participation in bundled payments is a natural source of heterogeneity across hospitals in our application. Using indications in the claims data regarding bundled payment eligibility, we find no evidence that bundled payments play a meaningful role in our overall estimates.
} 


\section{Hospital Influence on Physician Referrals}

Our results in Sections 5 and 6 show that integration tends to increase outpatient spending, consistent with Medicare location-based payment differentials, but that this increase is largely offset by a sizeable reduction in claims for professional services. The net effect is an economically small and statistically insignificant increase in total overall Medicare spending per episode.

While these are important findings from a policy-perspective, the results do not identify an underlying mechanism explaining the decrease in professional services claims. In the context of our theoretical framework in Section 2, the question is whether the reduction in services comes from a reduction in the physician's marginal revenue or from a change in the incentives associated with visits to other integrated versus non-integrated physicians. The former will be associated with a reduction in visits to the operating physician, while the latter will be associated with a reduction in visits to other non-integrated physicians outside of the hospital system. To address this question, we begin by identifying all providers visited within each episode and examining the relative frequency in which those providers are associated with the same hospital system. Specifically, we form an indicator set to 1 if the physician NPI in any given claim is part of a practice that is also owned by the same hospital system, and we count all such claims in the episode. We do this separately by institutional outpatient and physician services, and separately by whether the claim is associated with the same physician as the operating physician or with some other vertically integrated physician.

We present results in Table 6, where we find that the overall reduction in professional services claims comes entirely from non-integrated providers. Specifically, we estimate that vertical integration leads to a very modest increase of 0.18 claims going to the same operating physician, an increase of just over 3 claims to other vertically integrated providers, and a large decrease of 26 claims associated with other non-integrated providers. To aid the interpretation of these results, we also present estimates of the effects on payments (in dollars). These results show that the increase in claims to vertically integrated providers generates approximately $\$ 1,800$ in additional revenue for the acquiring hospital (assuming the hospital is the residual claimant on all such claims), which is more than offset by a reduction of $\$ 2,200$ in spending to non-integrated providers. We also estimate a reduction of \$240 in spending on professional services claims to the same physician, which is consistent with the mechanical reduction in Medicare payments when splitting payments across the physician fee schedule and the outpatient facility following integration.

These results show that patients are much more likely to stay "in the system" throughout the episode of care when the episode is initiated by a vertically integrated physician-hospital pair. This adjustment to referral patterns significantly increases the revenue for the vertically integrated system 
while similarly decreasing revenue to other non-integrated providers. Moreover, since the number of claims associated with the initiating physician remains largely unchanged, we conclude from this analysis that vertical integration does little to affect the average physician's own billable activity effort within an episode (i.e., on the intensive margin). In the context of our framework in Section 2, the replacement of $R_{j}\left(y_{i j k}\right)$ with $\bar{R}$ in the physician's optimization problem does not appear to have a major effect on patient treatment patterns conditional on a given surgery. Instead, changes to care within the episode derive almost entirely from reductions in visits to non-integrated providers. Combined with our reallocation results in Section 6, we conclude that vertical integration is a strong mechanism by which hospitals appear to gain influence over health care delivery within an episode.

As an additional analysis, we focus specifically on professional services claims in order to investigate the similarity between a recently acquired physician and their previously-acquired peers at the same hospital. The purpose of this analysis is to further assess the extent to which hospitals appear to influence physician behavior. While gauging such "similarity" is difficult with claims data, we measure similarity by comparing observed episodes of physicians at time $t$ to average episodes (for the same hospital) of integrated physicians in year $t-1$. To construct our outcome measure, we take the squared difference between the count of professional services claims for a given episode and the mean count among all episodes for physicians integrated with the same hospital in the prior year. The $\log$ of this squared difference is the outcome of interest. The key independent variable is again an indicator for whether the physician is integrated with hospital $k$, but in this case, we limit the analysis to physicians that were not integrated at time $t-1$. Therefore, at each time $t$, the sample consists

either of physicians that are not integrated with hospital $k$ or of physicians that are newly integrated with hospital $k$ in that year. We estimate a 95\% confidence interval of $[-1.04,-0.07]$, with a point estimate of -0.55 , for the effect of vertical integration on this measure of "similarity." In other words, newly-acquired physician practices become 50\% more similar (with regard to the count of professional services claims) to previously-acquired physicians compared to non-integrated physicians.

\section{Integration and Total Physician Effort}

As highlighted in Section 2, integration will tend remove a physician's individual profit motives and may therefore decrease the amount of care provided. From Table 6, we find no evidence that integration affects a physician's effort within an episode (i.e., on the intensive margin), as measured by the count of professional services claims. In this section, we broaden our measure of physician effort and estimate the effects of integration on total physician-level spending and number of claims. Our regression is therefore analogous to that in Equation 3, only at the physician level rather than the 
episode level. We again employ an instrumental variables estimation strategy, and we estimate the effects of integration on total billable activity (not limited to a $20 \%$ sample of patients) as well as effects on total professional services, inpatient, and outpatient services.

The results are summarized in Table 7 , where we estimate a large and statistically significant reduction in total billable activity per year. We estimate particularly large reductions (about 27\%) in professional services claims, which translates into reductions in spending of more than $\$ 49,000$. More interestingly, we estimate a reduction of around 4 inpatient surgeries per year among integrated physicians, accounting for around $\$ 100,000$ less revenue per year. ${ }^{19}$

\section{Discussion}

By vertically integrating with physician practices, hospitals and physicians become more financially integrated and to some degree share in the combined profits from hospital care and physician servicesthough not necessarily in a well-defined and easily contracted way. Compared to an independent or group practice not otherwise owned by a hospital system, vertical integration introduces potential pressure from the hospital system for a physician to change their treatment patterns to better align with the preferences and financial interests of the hospital. Some of these pressures may be purely cost reducing, such as adopting a standard medical device across physicians for a given procedure (Craig et al., 2021); however, other pressures may increase treatment intensity or reallocate care to different and higher reimbursing settings. Any such influences are also taking place against a backdrop of the newly formed employer-employee relationship, which can change physician incentives for billable activity effort, irrespective of hospital motivations or actions. The overall effect of these different and sometimes opposing forces is consequently an empirical question.

In this paper, we use Medicare FFS claims from a 20\% sample of Medicare beneficiaries to construct measures of spending and utilization for 90-day episodes of care. We focus our analysis on the effects of vertical integration between physicians and hospitals on episode-level spending and utilization. Our preferred results are those from an instrumental variables analysis in which we exploit a plausibly exogenous price shock to physician fee schedules in 2010 as an instrument for downstream physician-hospital vertical integration. Using our IV estimates, we estimate increases in Medicare spending for institutional outpatient claims alongside large reductions in total number of professional claims, which indicate less intensive treatment bundles, on average, after hospital-physician integration. On net, we find little evidence of any economically meaningful changes in Medicare spending

\footnotetext{
${ }^{19}$ We present analogous event study results following Callaway \& Sant'Anna (2020) in the supplemental appendix. These estimates are generally consistent with our preferred IV results, although the comparison is more nuanced relative to the other results in Sections 5, 6, and 7.
} 
per episode.

Turning specifically to the issue of hospital influence following the acquisition of their practice, we investigate changes in referral patterns for vertically integrated physicians. Here, we find strong evidence that vertical integration shapes referral patterns within an episode, wherein patients are much more likely to visit another integrated physician practice under common hospital ownership when the episode is initiated by a vertically integrated physician-hospital pair. This is consistent with hospitals exercising more influence over treatment patterns for additional financial gain-approximately $\$ 1,800$ on average in our analyses. The focal surgeons do not strongly increase or decrease their own billable activity per episode following hospital ownership, but instead, our evidence reveals that the reduction in professional service activity estimated in our main results entirely localizes to other, non-integrated physicians. Our findings are consistent with newly-integrated physicians conforming to the practice patterns of their already-integrated and less-intensive peers. The resulting reduction in episode treatment intensity consequently shields Medicare from the full spending increases that its payment policies would otherwise predict. That said, Medicare is simultaneously paying similar amounts over the 90-day window for beneficiaries to receive substantively less care, which implies a foregone savings opportunity for the public insurance program and taxpayers if a site-neutral payment regime were implemented. In other words, even if the services now absent from the episode post-integration were uniformly inefficient ("low-value") care, Medicare has effectively failed to capture any of the savings. At the same time, hospitals' inability to preserve the overall episode treatment intensity and redirect those additional services to its employed providers and owned assets (e.g., imaging and other diagnostic technology) means that these firms either choose not to or are unable to fully align physician behavior with a hospital revenue maximization objective. Beyond within-bundle treatment intensity changes, our estimates also reveal that the newly integrated surgeons schedule fewer elective surgeries and engage in less billable activity overall, which is at least consistent with weaker incentives for own effort after hospital employment.

Finally, we find no evidence of an effect of vertical integration on quality, measured using readmission rates, mortality rates, or incidence of complications following our clinical episodes of interest. Our key contribution is therefore to show the overall effect of hospital-physician integration on spending and utilization for well-defined treatment episodes, allowing for the combination of favorable reimbursement policy (which tends to increase Medicare payments following integration) as well as changes in care quantities due to the change in the physician's affiliation or employment with the hospital. Hospitals successfully capture new revenue streams by becoming the residual claimant on care provided by the newly owned physician and shifting some of their referrals toward other providers within the overarching firm. This is consistent with strategic behavior, rather than improved care 
delivery motivations, as the key driver of hospitals' acquisitions of physician practices. Our findings speak to contemporary Medicare payment policy debates and anticipated fiscal impacts, especially when the proposed reforms focus on site-of-care reimbursement rules. These findings, coupled with other known effects of vertical integration (e.g., increased bargaining power and higher prices), also encourage greater attention by regulators and antitrust authorities. 


\section{References}

Afendulis, Christopher C, \& Kessler, Daniel P. 2007. Tradeoffs from Integrating Diagnosis and Treatment in Markets for Health Care. American Economic Review, 1013-1020. Publisher: JSTOR.

Aron-Dine, Aviva, Einav, Liran, \& Finkelstein, Amy. 2013. The RAND Health Insurance Experiment, Three Decades Later. Journal of Economic Perspectives, 27(1), 197-222. Publisher: American Economic Association.

Baicker, Katherine, \& Chandra, Amitabh. 2004. Medicare Spending, The Physician Workforce, And Beneficiaries' Quality Of Care. Health Affairs, 23(3), 291.

Baker, Laurence C, Bundorf, M Kate, \& Kessler, Daniel P. 2014. Vertical integration: hospital ownership of physician practices is associated with higher prices and spending. Health Affairs, 33(5), 756-763. Publisher: Health Affairs.

Baker, Laurence C, Bundorf, M Kate, \& Kessler, Daniel P. 2016. The Effect of Hospital/Physician Integration on Hospital Choice. Journal of Health Economics, 50, 1-8.

Borgen, N. T. 2016. Fixed effects in unconditional quantile regression. Stata Journal, 16(2), 403415(13). Place: College Station, TX Publisher: Stata Press.

Callaway, Brantly, \& Sant'Anna, Pedro HC. 2020. Difference-in-differences with multiple time periods. Journal of Econometrics.

Capps, Cory, Dranove, David, \& Ody, Christopher. 2018. The effect of hospital acquisitions of physician practices on prices and spending. Journal of health economics, 59, 139-152. Publisher: Elsevier.

Carlin, Caroline S, Dowd, Bryan, \& Feldman, Roger. 2015a. Changes in quality of health care delivery after vertical integration. Health services research, 50(4), 1043-1068. Publisher: Wiley Online Library.

Carlin, Caroline S, Feldman, Roger, \& Dowd, Bryan. 2015b. The Impact of Hospital Acquisition of Physician Practices on Referral Patterns. Health economics. Publisher: Wiley Online Library.

Chernew, Michael, Cooper, Zack, Larsen Hallock, Eugene, \& Scott Morton, Fiona. 2021. Physician Agency, Consumerism, and the Consumption of Lower-Limb MRI Scans. Journal of Health Economics, 76, online ahead of print. Publisher: Elsevier. 
Ciliberto, Federico, \& Dranove, David. 2006. The effect of physician-hospital affiliations on hospital prices in California. Journal of Health Economics, 25(1), 29-38. Publisher: Elsevier.

Clemens, Jeffrey, \& Gottlieb, Joshua D. 2014. Do Physicians' Financial Incentives Affect Medical Treatment and Patient Health? American Economic Review, 104(4), 1320-1349.

Craig, Stuart V., Grennan, Matthew, \& Swanson, Ashley. 2021. Mergers and marginal costs: New evidence on hospital buyer power. The RAND Journal of Economics, 52(1), 151-178. _eprint: https://onlinelibrary.wiley.com/doi/pdf/10.1111/1756-2171.12365.

Cuellar, Alison Evans, \& Gertler, Paul J. 2006. Strategic integration of hospitals and physicians. Journal of Health Economics, 25(1), 1-28. Publisher: Elsevier.

Cuesta, José Ignacio, Noton, Carlos, \& Vatter, Benjamin. 2019. Vertical integration between hospitals and insurers. Available at SSRN 3309218.

Dranove, David, \& Ody, Christopher. 2019. Employed for higher pay? How Medicare facility fees affect hospital employment of physicians. American Economics Journal: Economic Policy, 11(4), 249-271.

Dummit, Laura A, Kahvecioglu, Daver, Marrufo, Grecia, Rajkumar, Rahul, Marshall, Jaclyn, Tan, Eleonora, Press, Matthew J, Flood, Shannon, Muldoon, L Daniel, Gu, Qian, \& others. 2016. Association between hospital participation in a Medicare bundled payment initiative and payments and quality outcomes for lower extremity joint replacement episodes. Jama, 316(12), 1267-1278. Publisher: American Medical Association.

Ellimoottil, Chad, Ryan, Andrew M, Hou, Hechuan, Dupree, James M, Hallstrom, Brian, \& Miller, David C. 2017. Implications of the definition of an episode of care used in the comprehensive care for joint replacement model. JAMA surgery, 152(1), 49-54. Publisher: American Medical Association.

Ellis, Randall P, \& McGuire, Thomas G. 1996. Hospital response to prospective payment: moral hazard, selection, and practice-style effects. Journal of health economics, 15(3), 257-277. Publisher: Elsevier.

Finkelstein, Amy, \& McGarry, Kathleen. 2006. Multiple dimensions of private information: evidence from the long-term care insurance market. American Economic Review, 96(4), 938-958. Publisher: Princeton, NJ: American Economic Association, 1911-. 
Finkelstein, Amy, Gentzkow, Matthew, \& Williams, Heidi. 2016. Sources of geographic variation in health care: Evidence from patient migration. The quarterly journal of economics, 131(4), 16811726.

Firpo, Sergio, Fortin, Nicole M, \& Lemieux, Thomas. 2009. Unconditional quantile regressions. Econometrica, 77(3), 953-973. Publisher: Wiley Online Library.

Gaynor, M, \& Town, R. 2012. Competition in Health Care Markets. In: Pauly, M., McGuire, T., \& Pita Barros, P. (eds), Handbook of Health Economics, vol. 2. Amsterdam: Elsevier.

Gaynor, M, Ho, K, \& Town, R. 2015. The Industrial Organization of Health Care Markets. Journal of Economic Literature, 47(2), 235-284.

Gaynor, Martin, \& Gertler, Paul. 1995. Moral hazard and risk spreading in partnerships. The RAND Journal of Economics, 591-613. Publisher: JSTOR.

Gottlieb, Daniel J, Zhou, Weiping, Song, Yunjie, Andrews, Kathryn Gilman, Skinner, Jonathan S, \& Sutherland, Jason M. 2010. Prices don't drive regional Medicare spending variations. Health Affairs, 29(3), 537-543.

Gowrisankaran, Gautam, Nevo, Aviv, \& Town, Robert. 2015. Mergers When Prices Are Negotiated: Evidence from the Hospital Industry. American Economic Review, 105(1), 172-203.

Gruber, Jonathan, \& Owings, Maria. 1996. Physician financial incentives and cesarean section delivery. The RAND Journal of Economics, 27(1), 99-123.

Ho, Kate, \& Lee, Robin S. 2017. Insurer competition in health care markets. Econometrica, 85(2), 379-417. Publisher: Wiley Online Library.

Iizuka, Toshiaki. 2012. Physician agency and adoption of generic pharmaceuticals. American Economic Review, 102(6), 2826-58.

Koch, Thomas G, Wendling, Brett W, \& Wilson, Nathan E. 2017. How vertical integration affects the quantity and cost of care for Medicare beneficiaries. Journal of Health Economics, 52, 19-32. Publisher: Elsevier.

Koch, Thomas G, Wendling, Brett W, \& Wilson, Nathan E. 2018. The Effects of Physician and Hospital Integration on Medicare Beneficiaries' Health Outcomes. Review of Economics and Statistics, 1-38. Publisher: MIT Press. 
Kocher, Robert, \& Sahni, Nikhil R. 2011. Hospitals' race to employ physicians-the logic behind a money-losing proposition. New England Journal of Medicine, 364(19), 1790-1793. Publisher: Mass Medical Soc.

Lin, Haizhen, McCarthy, Ian M, \& Richards, Michael. 2021. Hospital Pricing Following Integration with Physician Practices. Journal of Health Economics.

Madison, Kristin. 2004. Hospital-physician affiliations and patient treatments, expenditures, and outcomes. Health services research, 39(2), 257-278. Publisher: Wiley Online Library.

Mechanic, Robert E. 2015. Mandatory Medicare bundled payment-is it ready for prime time? New England Journal of Medicine, 373(14), 1291-1293. Publisher: Mass Medical Soc.

Molitor, David. 2018. The evolution of physician practice styles: evidence from cardiologist migration. American Economic Journal: Economic Policy, 10(1), 326-56.

Post, Brady, Buchmueller, Tom, \& Ryan, Andrew M. 2018. Vertical integration of hospitals and physicians: Economic theory and empirical evidence on spending and quality. Medical Care Research and Review, 75(4), 399-433. Publisher: SAGE Publications Sage CA: Los Angeles, CA.

Richards, Michael R., Seward, Jonathan A., \& Whaley, Christopher M. 2022. Treatment consolidation after vertical integration: Evidence from outpatient procedure markets. Journal of Health Economics, 81(Jan.), 102569.

Skinner, Jonathan, \& Fisher, Elliott. 1997. Regional disparities in Medicare expenditures: an opportunity for reform. National Tax Journal, 50(3), 413-425.

Starc, Amanda, \& Town, Robert J. 2020. Externalities and Benefit Design in Health Insurance. The Review of Economic Studies, 87(6), 2827-2858.

Walden, Emily. 2016. Can Hospitals Buy Referrals? The Impact of Physician Group Acquisitions on Market-Wide Referral Patterns. Working Paper. University of Wisconsin, Madison.

Wennberg, John, \& Gittelsohn, Alan. 1973. Small area variations in health care delivery: a populationbased health information system can guide planning and regulatory decision-making. Science, 182(4117), 1102-1108.

Wennberg, John E, Fisher, Elliott S, \& Skinner, Jonathan S. 2003. Geography and the debate over medicare reform. Health Affairs, W96. 
Whaley, Christopher M, Zhao, Xiaoxi, Richards, Michael, \& Damberg, Cheryl L. 2021. Higher Medicare Spending on Imaging and Lab Services after Primary Care Physician Group Vertical Integration. Health Affairs, 40(5), 702-709.

Young, Gary J, Zepeda, E David, Flaherty, Stephen, \& Thai, Ngoc. 2021. Hospital Employment of Physicians in Massachusetts Is Associated with Inappropriate Diagnostic Imaging. Health Affairs, 40(5), 710-718. Publisher: Health Affairs. 


\section{Tables and Figures}

Figure 1: Top 10 DRGs ${ }^{a}$

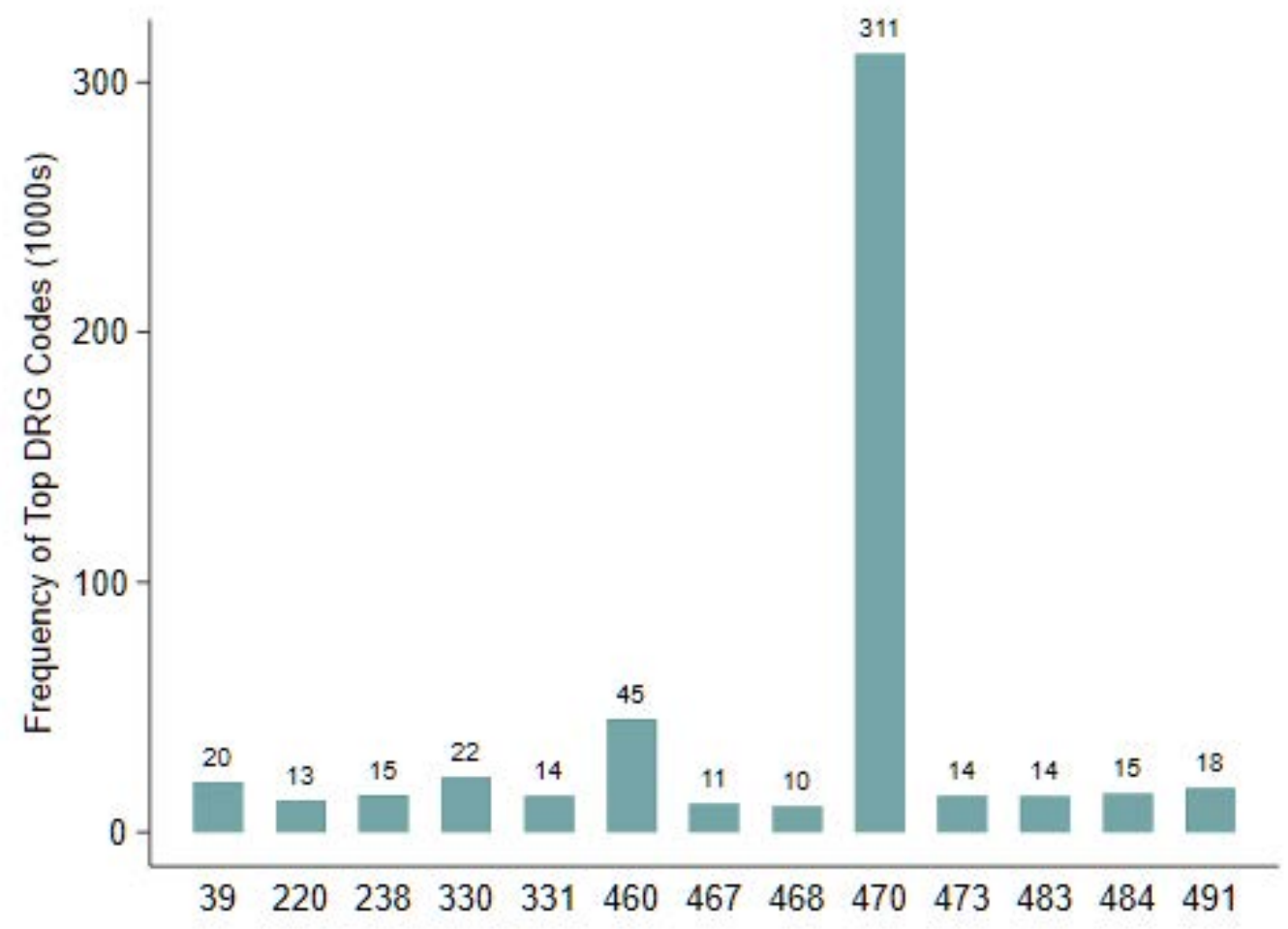

${ }^{a}$ Frequency of most common DRGs associated with the initial inpatient stay of an episode. For a comprehensive list of DRGs and descriptions, see http://data.nber.org/drg/drgdesc11.pdf. 
Figure 2: Event Studies ${ }^{a}$

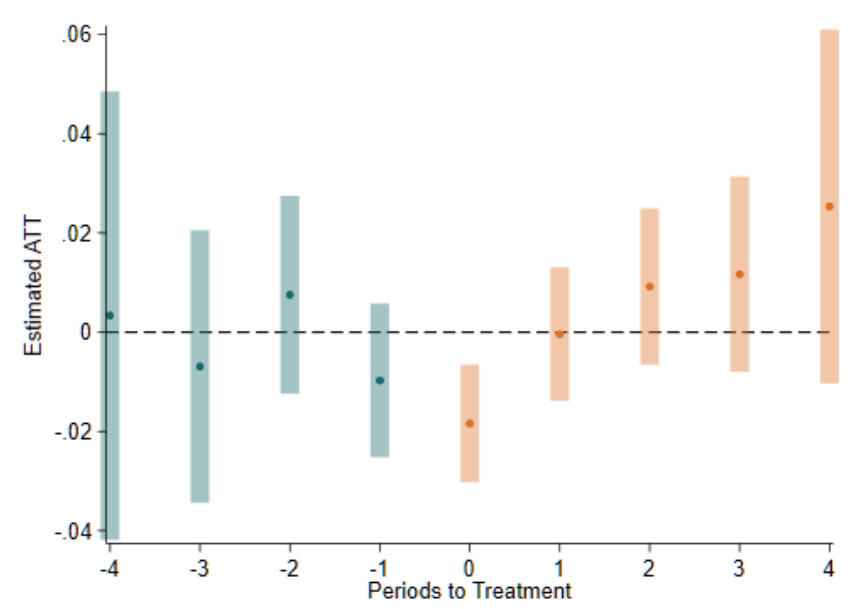

a. Episode Payments

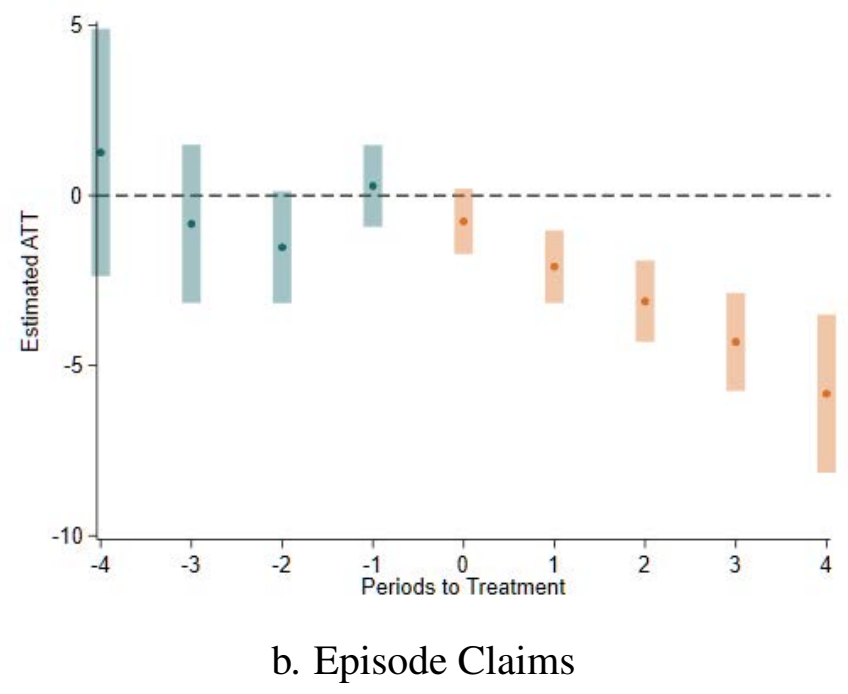

${ }^{a}$ Figures present the results of yearly ATTs based on the repeated cross-section version of Callaway \& Sant'Anna (2020), without adjustment for hospital-physician fixed effects and without imposing balance in the hospital-physician panel. Period 0 denotes the first year of integration. 
Figure 3: Physician-level PPIS Relative Revenue Change ${ }^{a}$

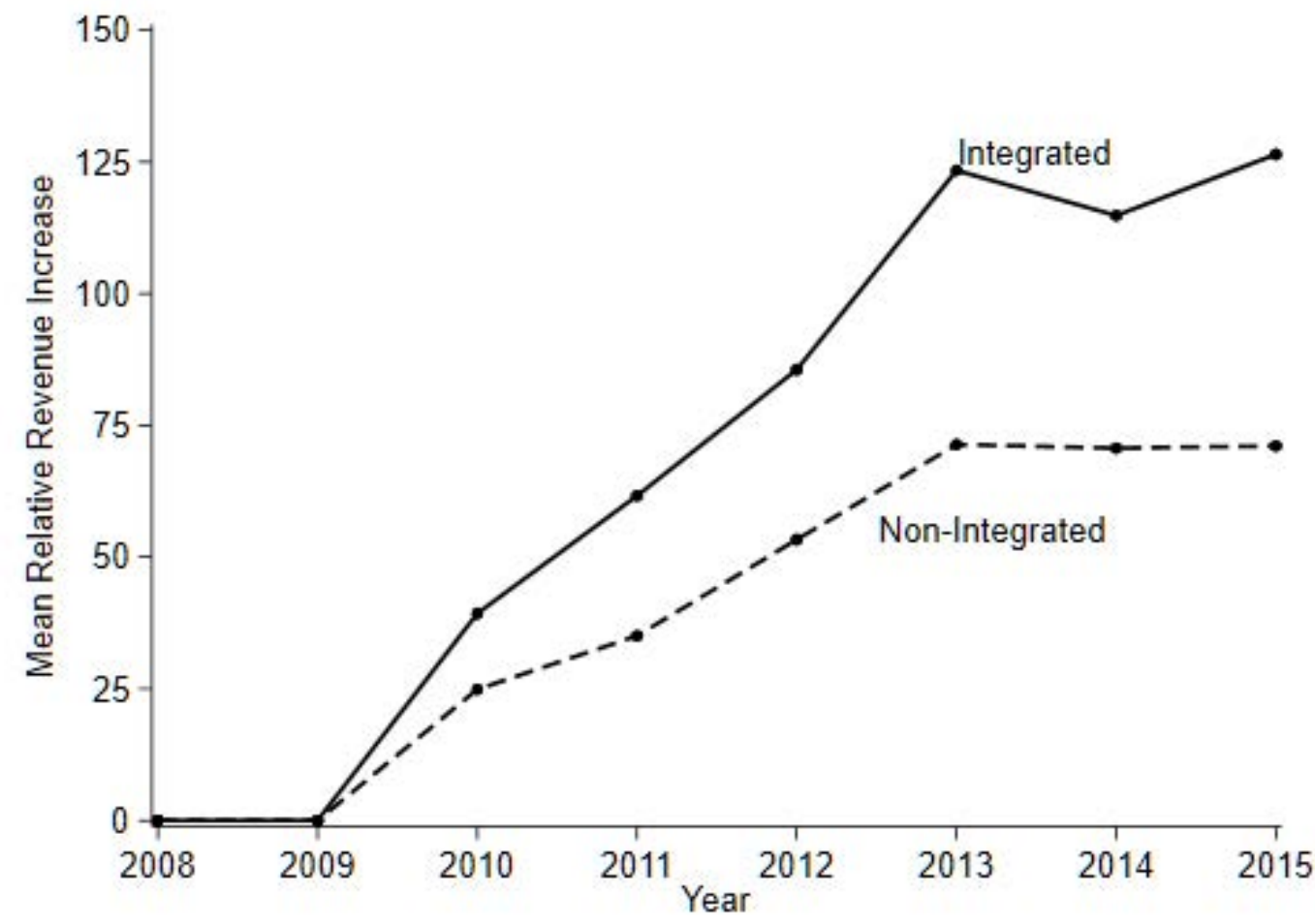

${ }^{a}$ Mean revenue change calculated from Equation 4 in the text. "Integrated" denotes physicians that are vertically integrated at some point over our panel, and "non-integrated" denotes physicians that are never integrated in our data. 
Figure 4: Estimates from LPM of Vertical Integration ${ }^{a}$

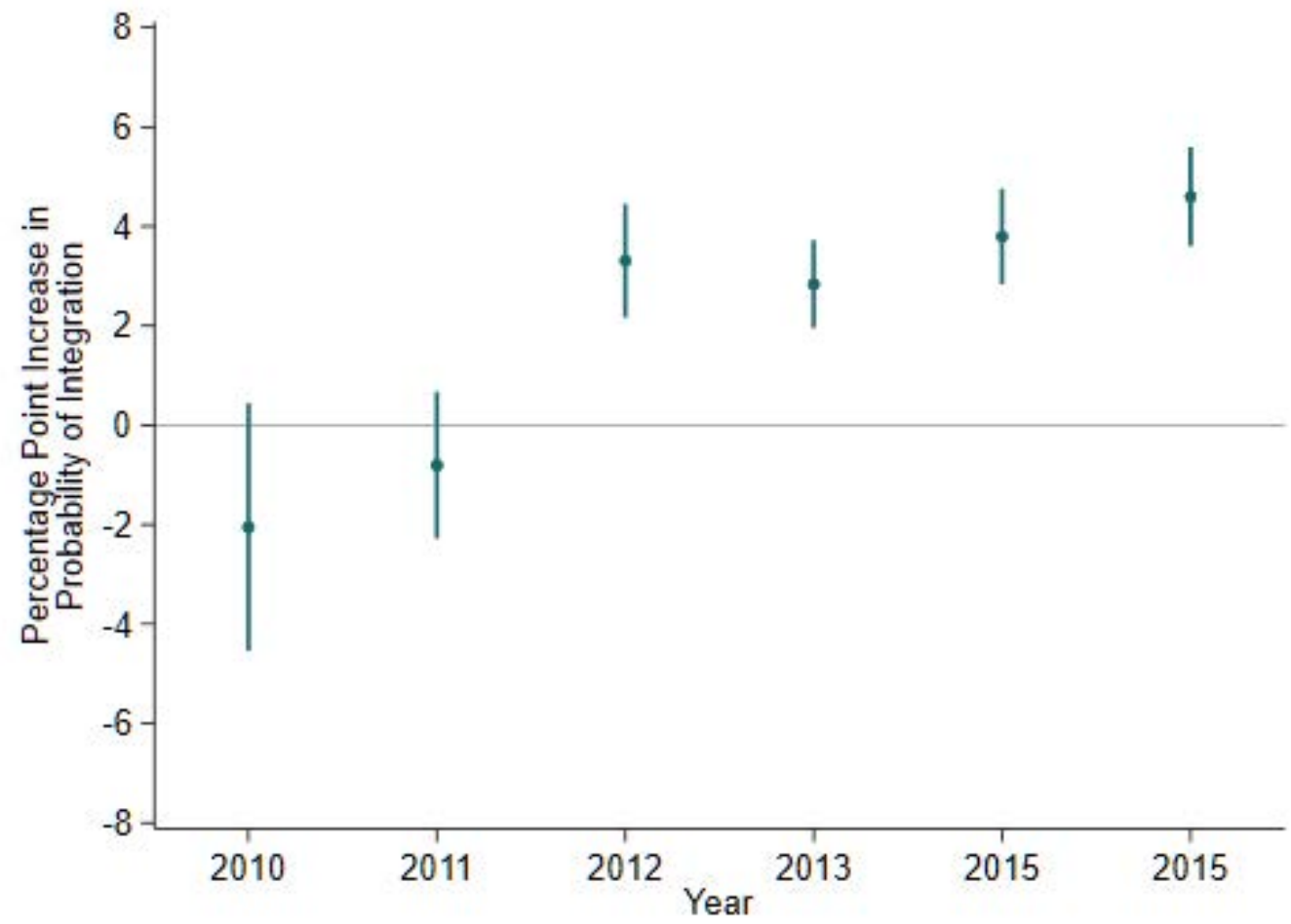

${ }^{a}$ Point estimates and $95 \%$ confidence intervals for the coefficients on $\Delta$ Revenue $_{j, t}^{2010} \times$ $1(t)$ based on a linear regression of physician integration on $\Delta$ Revenue $_{j, t}^{2007}$, $\Delta$ Revenue $_{j, t}^{2010}$, year dummies, and interactions of year dummies on the revenue change variables, $\Delta$ Revenue $_{j, t}^{2007} \times 1(t)$ and $\Delta$ Revenue $_{j, t}^{2010} \times 1(t)$. The revenue change variables are defined explicitly in Equation 4. 


\section{Figure 5: Specification Curves for IV Estimates $^{a}$}



a. Episode payments

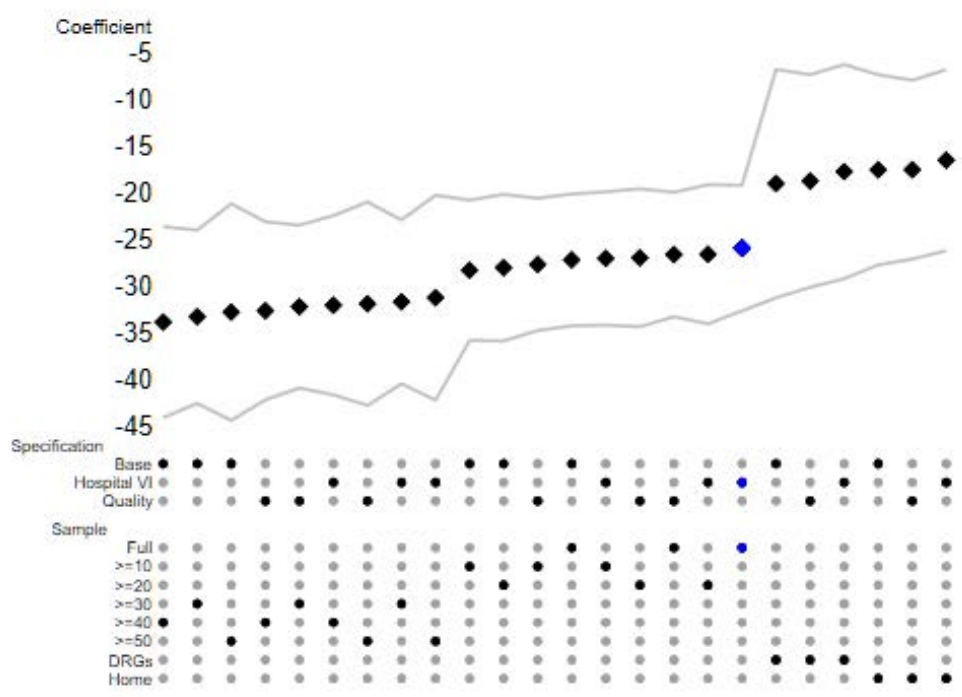

b. Number of claims

\footnotetext{
${ }^{a}$ Point estimates and $95 \%$ confidence intervals for the coefficient on physician-hospital vertical integration estimated using IV. "Base" denotes a specification without adjusting for hospital-level vertical integration or quality of care in an episode; "Hospital VI" denotes a specification that includes an indicator for hospital-level vertical integration (the preferred specification presented in the text); and "Quality" denotes a specification that includes an indicator for within-episode mortality as an additional control variable. "Full" denotes the full sample, which is the sample underlying the results in the main text; " $>=10$ " through " $>=50$ " denote restrictions on the minimum number of episodes per physician-hospital pair in the estimation sample; "DRGs" denotes the sample that is restricted to orthopedic procedures only; and "Home" denotes a sample that is restricted to patients discharged to home. The code for our specification curves was adapted from Hans S. Sievertson, who kindly made his code available at github.com/hhsievertsen/speccurve.
} 
Figure 6: Quantile Regression Results for Total Payments and Claims ${ }^{a}$
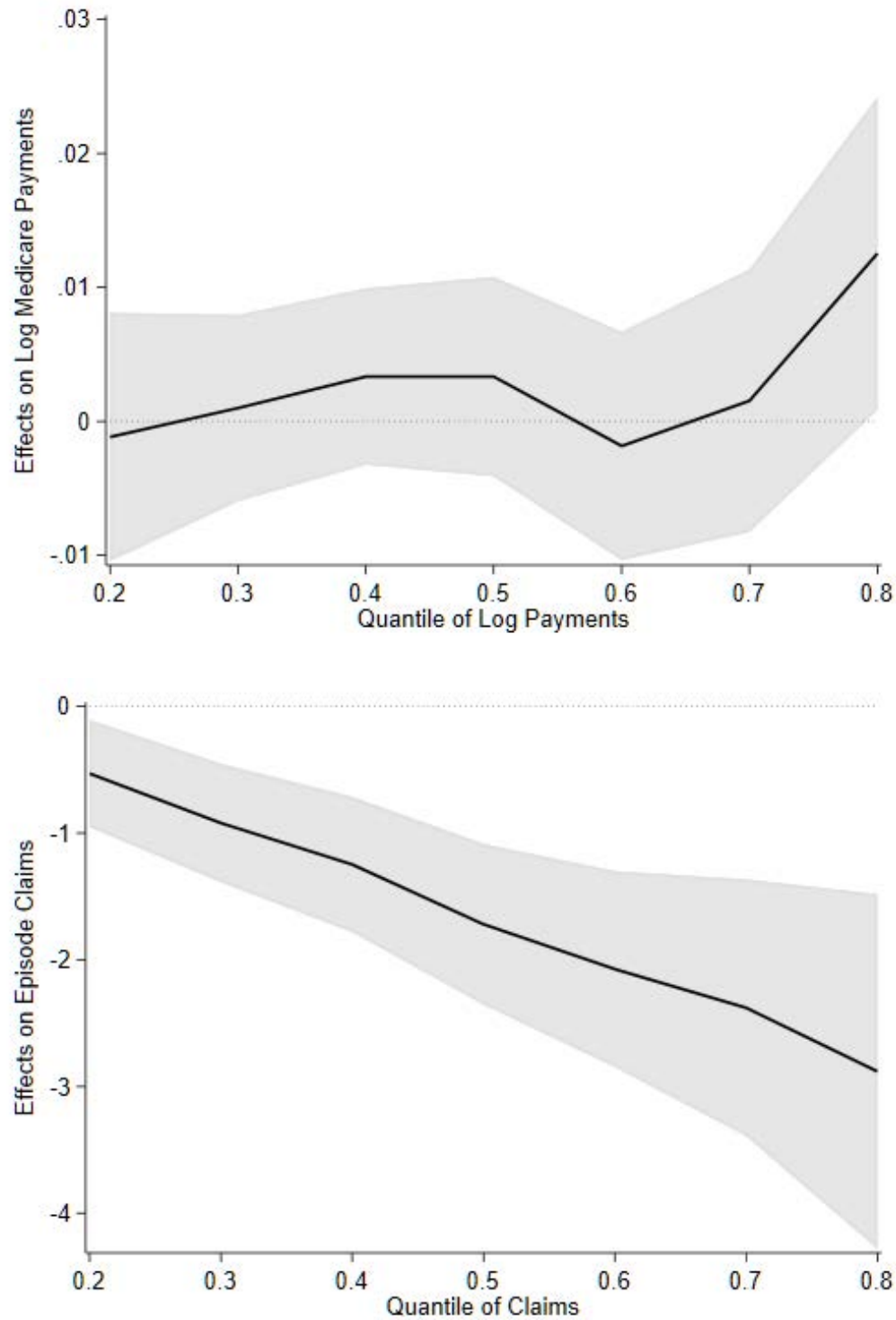

${ }^{a}$ Estimates and $95 \%$ confidence intervals for the coefficient on vertical integration from unconditional quantile regressions of log Medicare payments and number of claims (Firpo et al., 2009; Borgen, 2016). 
Table 1: Descriptive Statistics per Episode ${ }^{a}$

\begin{tabular}{|c|c|c|c|c|c|c|c|}
\hline & 2010 & 2011 & 2012 & 2013 & 2014 & 2015 & Total \\
\hline \multirow[t]{2}{*}{ Medicare Payments (1000s) } & 23.835 & 24.404 & 25.000 & 25.523 & 26.411 & 26.609 & 25.181 \\
\hline & $(17.109)$ & $(17.361)$ & $(17.591)$ & $(17.455)$ & $(17.687)$ & $(17.807)$ & $(17.505)$ \\
\hline \multirow[t]{2}{*}{ Log Payments } & 9.847 & 9.872 & 9.898 & 9.934 & 9.990 & 10.000 & 9.917 \\
\hline & $(0.712)$ & $(0.712)$ & $(0.718)$ & $(0.692)$ & $(0.635)$ & $(0.626)$ & $(0.690)$ \\
\hline \multirow[t]{2}{*}{ Claims } & 70.833 & 69.408 & 73.162 & 75.029 & 76.480 & 73.738 & 72.969 \\
\hline & $(62.338)$ & $(61.505)$ & $(60.955)$ & $(60.972)$ & $(61.432)$ & $(58.975)$ & $(61.214)$ \\
\hline \multirow[t]{2}{*}{ Mortality } & 0.038 & 0.036 & 0.037 & 0.034 & 0.032 & 0.031 & 0.035 \\
\hline & $(0.191)$ & $(0.187)$ & $(0.188)$ & $(0.181)$ & $(0.177)$ & $(0.173)$ & $(0.184)$ \\
\hline \multirow[t]{2}{*}{ Readmission } & 0.177 & 0.173 & 0.165 & 0.156 & 0.153 & 0.150 & 0.164 \\
\hline & $(0.382)$ & $(0.378)$ & $(0.372)$ & $(0.363)$ & $(0.360)$ & $(0.357)$ & $(0.370)$ \\
\hline \multirow[t]{2}{*}{ Sepsis } & 0.021 & 0.021 & 0.021 & 0.021 & 0.023 & 0.020 & 0.021 \\
\hline & $(0.142)$ & $(0.143)$ & $(0.144)$ & $(0.145)$ & $(0.149)$ & $(0.140)$ & $(0.144)$ \\
\hline \multirow[t]{2}{*}{ SSI } & 0.017 & 0.017 & 0.016 & 0.016 & 0.016 & 0.013 & 0.016 \\
\hline & $(0.128)$ & $(0.129)$ & $(0.125)$ & $(0.125)$ & $(0.127)$ & $(0.115)$ & $(0.126)$ \\
\hline \multirow[t]{2}{*}{ Any Complication } & 0.035 & 0.035 & 0.035 & 0.035 & 0.036 & 0.031 & 0.035 \\
\hline & $(0.184)$ & $(0.184)$ & $(0.183)$ & $(0.183)$ & $(0.187)$ & $(0.174)$ & $(0.183)$ \\
\hline \multirow[t]{2}{*}{ Integrated } & 0.119 & 0.176 & 0.286 & 0.324 & 0.356 & 0.369 & 0.262 \\
\hline & $(0.323)$ & $(0.381)$ & $(0.452)$ & $(0.468)$ & $(0.479)$ & $(0.483)$ & $(0.440)$ \\
\hline Observations & 192,049 & 189,931 & 182,982 & 176,361 & 166,843 & 121,865 & $, 030,031$ \\
\hline
\end{tabular}

${ }^{a}$ Mean values by year calculated across all episodes, with standard deviations in parenthesis. In order to allow for a full 90-day episode, we exclude all episodes initiated after September 2015. 
Table 2: Descriptive Statistics per Physician $^{a}$

\begin{tabular}{l|rrrrrrr} 
& \multicolumn{1}{c}{2010} & \multicolumn{1}{c}{2011} & \multicolumn{1}{c}{2012} & \multicolumn{1}{c}{2013} & \multicolumn{1}{c}{2014} & \multicolumn{1}{c}{2015} & \multicolumn{1}{c}{ Total } \\
\hline Episodes & 4.913 & 4.974 & 5.019 & 5.067 & 5.113 & 4.200 & 4.901 \\
& $(5.995)$ & $(6.180)$ & $(6.288)$ & $(6.466)$ & $(6.528)$ & $(5.210)$ & $(6.152)$ \\
Practice Size & 11.415 & 12.520 & 13.009 & 12.593 & 12.314 & 11.945 & 12.338 \\
& $(30.561)$ & $(33.686)$ & $(36.591)$ & $(33.671)$ & $(33.365)$ & $(32.068)$ & $(33.510)$ \\
Experience & 22.340 & 23.224 & 23.047 & 23.815 & 23.706 & 24.763 & 23.476 \\
& $(6.279)$ & $(6.432)$ & $(6.434)$ & $(6.599)$ & $(6.637)$ & $(6.635)$ & $(6.544)$ \\
Female & 0.100 & 0.109 & 0.105 & 0.109 & 0.104 & 0.106 & 0.106 \\
& $(0.193)$ & $(0.195)$ & $(0.192)$ & $(0.191)$ & $(0.183)$ & $(0.184)$ & $(0.190)$ \\
Includes PCP & 0.028 & 0.028 & 0.026 & 0.021 & 0.019 & 0.016 & 0.023 \\
& $(0.164)$ & $(0.166)$ & $(0.160)$ & $(0.143)$ & $(0.135)$ & $(0.124)$ & $(0.150)$ \\
Multi-specialty & 0.274 & 0.221 & 0.262 & 0.276 & 0.369 & 0.374 & 0.292 \\
Surgery Center & $(0.446)$ & $(0.415)$ & $(0.440)$ & $(0.447)$ & $(0.483)$ & $(0.484)$ & $(0.455)$ \\
& 0.506 & 0.545 & 0.531 & 0.547 & 0.552 & 0.482 & 0.529 \\
Integrated & $(0.500)$ & $(0.498)$ & $(0.499)$ & $(0.498)$ & $(0.497)$ & $(0.500)$ & $(0.499)$ \\
& 0.125 & 0.191 & 0.307 & 0.355 & 0.386 & 0.396 & 0.285 \\
\hline Observations & $(0.330)$ & $(0.393)$ & $(0.461)$ & $(0.478)$ & $(0.487)$ & $(0.489)$ & $(0.451)$ \\
\hline
\end{tabular}

${ }^{a}$ Mean values by year calculated across unique physicians, with standard deviations in parenthesis. In order to allow for a full 90-day episode, we exclude all episodes initiated after September 2015. 
Table 3: Descriptive Statistics per Hospital ${ }^{a}$

\begin{tabular}{l|rrrrrrr} 
& \multicolumn{1}{|c}{2010} & \multicolumn{1}{c}{2011} & \multicolumn{1}{c}{2012} & \multicolumn{1}{c}{2013} & 2014 & 2015 & \multicolumn{1}{c}{ Total } \\
\hline Episodes & 55.666 & 55.633 & 53.708 & 52.101 & 50.497 & 38.528 & 51.187 \\
& $(78.225)$ & $(78.154)$ & $(75.546)$ & $(72.952)$ & $(71.189)$ & $(54.159)$ & $(72.609)$ \\
Nurse FTEs (100s) & 3.895 & 3.973 & 4.081 & 4.234 & 4.401 & 4.642 & 4.194 \\
& $(4.725)$ & $(4.939)$ & $(5.111)$ & $(5.324)$ & $(5.501)$ & $(5.822)$ & $(5.241)$ \\
Other FTEs (100s) & 8.248 & 8.394 & 8.520 & 8.716 & 8.845 & 9.270 & 8.652 \\
& $(9.996)$ & $(10.585)$ & $(10.898)$ & $(11.230)$ & $(11.382)$ & $(11.897)$ & $(10.995)$ \\
Beds (100s) & 2.146 & 2.157 & 2.155 & 2.191 & 2.210 & 2.269 & 2.186 \\
& $(2.101)$ & $(2.123)$ & $(2.161)$ & $(2.215)$ & $(2.241)$ & $(2.318)$ & $(2.191)$ \\
For-profit & 0.156 & 0.167 & 0.157 & 0.158 & 0.158 & 0.162 & 0.160 \\
& $(0.363)$ & $(0.373)$ & $(0.364)$ & $(0.365)$ & $(0.364)$ & $(0.368)$ & $(0.366)$ \\
System Affiliation & 0.600 & 0.622 & 0.643 & 0.655 & 0.674 & 0.692 & 0.646 \\
& $(0.490)$ & $(0.485)$ & $(0.479)$ & $(0.475)$ & $(0.469)$ & $(0.462)$ & $(0.478)$ \\
Major Teaching & 0.069 & 0.072 & 0.067 & 0.069 & 0.065 & 0.070 & 0.069 \\
& $(0.254)$ & $(0.258)$ & $(0.250)$ & $(0.254)$ & $(0.246)$ & $(0.254)$ & $(0.253)$ \\
Integrated & 0.377 & 0.441 & 0.584 & 0.633 & 0.656 & 0.654 & 0.555 \\
& $(0.485)$ & $(0.497)$ & $(0.493)$ & $(0.482)$ & $(0.475)$ & $(0.476)$ & $(0.497)$ \\
\hline Observations & 3,450 & 3,414 & 3,407 & 3,385 & 3,304 & 3,163 & 20,123
\end{tabular}

${ }^{a}$ Mean values by year calculated across unique hospitals, with standard deviations in parenthesis. In order to allow for a full 90-day episode, we exclude all episodes initiated after September 2015. 
Table 4: Instrumental Variables Estimates ${ }^{a}$

\begin{tabular}{l|rr} 
& Log Payments & Number of Claims \\
\hline Integrated with Hospital $k$ & 0.023 & $-25.903 * * *$ \\
& $(0.030)$ & $(3.428)$ \\
Any Hospital Integration & -0.005 & $1.842^{* * *}$ \\
\multicolumn{1}{l|}{} & $(0.003)$ & $(0.380)$ \\
\hline Hospital Characteristics & -0.000 & -0.001 \\
\hline Nurse FTEs & $(0.000)$ & $(0.001)$ \\
Other FTEs & $0.000^{*}$ & $0.001 * * *$ \\
& $(0.000)$ & $(0.000)$ \\
Beds (100s) & 0.000 & 0.161 \\
& $(0.002)$ & $(0.252)$ \\
For-profit & -0.004 & -3.507 \\
& $(0.022)$ & $(2.206)$ \\
System Affiliation & 0.003 & $-0.939 *$ \\
& $(0.005)$ & $(0.553)$ \\
Major Teaching & 0.002 & $-1.907 *$ \\
& $(0.009)$ & $(1.011)$ \\
\hline Observations & 746,744 & 731,815 \\
& &
\end{tabular}

${ }^{a}$ Results from instrumental variables with hospital-physician, year, and month fixed effects. Standard errors are in parenthesis, clustered by physician. We instrument for the vertical integration indicator as discussed in Section 4. All regressions include county demographic variables (total population, age distribution, income distribution, and education), dummies for whether the county hospital market is a monopoly, duopoly, or triopoly, as well as indicators for the DRG code of the initial inpatient stay and dummies for the quantile of the beneficiary's prior health care utilization. ${ }^{*}$ p-value $<0.1$, ** p-value $<0.05$, *** p-value $<0.01$ 
Table 5: Effects on Components of an Episode ${ }^{a}$

\begin{tabular}{c|rr} 
& Payments $(\$)$ & Claims \\
\hline Episode $^{b}$ & $1,171.07$ & $-25.903 * * *$ \\
& $(842.924)$ & $(3.428)$ \\
Inpatient $^{c}$ & 101.775 & 0.001 \\
& $(251.862)$ & $(0.018)$ \\
Outpatient & $307.932 * *$ & 0.407 \\
& $(155.196)$ & $(0.264)$ \\
Professional & -338.264 & $-22.936 * * *$ \\
& $(216.950)$ & $(3.159)$ \\
SNF & -139.712 & -0.023 \\
& $(130.571)$ & $(0.017)$ \\
Home Health & $131.257 * *$ & 0.017 \\
& $(74.171)$ & $(0.022)$
\end{tabular}

${ }^{a}$ IV results for the coefficient on hospital-physician vertical integration. We exclude, separately for each outcome, observations in the upper $1 \%$ of values, and we instrument for the vertical integration indicator as discussed in Section 4. Included covariates and fixed effects follow from Table 4. Standard errors in parentheses, clustered by physician. * p-value $<0.1$, ** p-value $<0.05$, *** p-value $<0.01$

${ }^{b}$ Since we examine each outcome separately, the samples underlying each estimate differ slightly across outcomes, and the estimates for each component therefore need not add to the total.

${ }^{c}$ Only includes claims in the inpatient setting incurred after the initial inpatient stay. 
Table 6: Effects on Referral Patterns ${ }^{a}$

\begin{tabular}{l|rr} 
& Payments $(\$)$ & Claims \\
\hline Same Physician & $-239.51^{* * *}$ & 0.179 \\
& $(65.682)$ & $(0.114)$ \\
Other VI Physicians & $1,804.73 * * *$ & $3.264 * * *$ \\
& $(57.813)$ & $(0.148)$ \\
Other Non-VI Physicians & $-2,200.96 * *$ & $-26.371^{* * *}$ \\
& $(218.083)$ & $(3.245)$
\end{tabular}

${ }^{a}$ IV results for the coefficient on hospital-physician vertical integration. We exclude, separately for each outcome, observations in the upper $1 \%$ of values, and we instrument for the vertical integration indicator as discussed in Section 4. Outcomes derive from all professional services claims within an episode. Standard errors in parentheses, clustered by physician. Included covariates and fixed effects follow from Table 4 . * p-value $<0.1, * *$ p-value $<0.05, * * *$ p-value $<0.01$ 
Table 7: Effects on Physician Effort ${ }^{a}$

\begin{tabular}{r|rr} 
& Payments $(\$)$ & Claims \\
\hline Total $^{b}$ & $-49,541^{* * *}$ & $-579.340 * * *$ \\
& $(27,907)$ & $(76.856)$ \\
Mean & {$[725,769]$} & {$[2,190]$} \\
\hline Professional Services & 1,257 & $-570.143 * * *$ \\
& $(4,423)$ & $(75.118)$ \\
Mean & {$[172,736]$} & {$[2,045]$} \\
\hline Inpatient Claims & $-100,225^{* * *}$ & $-4.110^{* * *}$ \\
& $(22,196)$ & $(1.271)$ \\
Mean & {$[380,243]$} & {$[27.380]$} \\
\hline Outpatient Claims & $14,256^{* *}$ & $9.529 *$ \\
& $(7,021)$ & $(5.162)$ \\
Mean & {$[114,822]$} & {$[75.414]$}
\end{tabular}

${ }^{a} \mathrm{IV}$ results for the coefficient on physician-level vertical integration. We exclude, separately for each outcome, observations in the upper and lower $5 \%$ of values, and we instrument for the vertical integration indicator as discussed in Section 4. Outcomes consist of all claims on which the operating physician is listed as the billing physician. Covariates include all county-level variables and mean patient-level variables described in Section 4, as well as physician fixed effects, with standard errors clustered by physician. Mean outcome levels in brackets. $*$ p-value $<0.1$, ** p-value $<0.05$, *** p-value $<0.01$

${ }^{b}$ Since we examine each outcome separately, the samples underlying each estimate differ slightly across outcomes, and the estimates for each component therefore need not add to the total. 